\title{
Observation of Constraint Surface Dynamics of Polystyrene Thin Films by Functionalization of a Silsesquioxane Cage
}

Taiki Hoshino $^{1,2}$, Shiki Nojima ${ }^{1,3}$, Masanao Sato ${ }^{3}$, Tomoyasu Hirai ${ }^{1,3,4,5}$, Yuji Higaki ${ }^{1,3,4,5,6}$, So Fujinami ${ }^{1}$, Daiki Murakami, Shigesaburo Ogawa, Hiroshi Jinnai ${ }^{6}{ }^{6,7}$, Atsushi Takahara $^{1,3,4,5,6}$, Masaki Takata ${ }^{1,7}$

${ }^{1}$ RIKEN, SPring-8 Center, 1-1-1 Kouto, Sayo-cho, Sayo-gun, Hyogo 679-5148, Japan

${ }^{2}$ JST-PRESTO, Tokyo Institute of Technology, 4259 Nagatsuta, Midori-ku, Yokohama, Kanagawa 226-8502, Japan

${ }^{3}$ Graduate School of Engineering, Kyushu University, Japan

${ }^{4}$ Institute for Materials Chemistry and Engineering, Kyushu University, 744 Motooka, Nishi-ku, Fukuoka 819-0395, Japan

${ }^{5}$ International Institute for Carbon-Neutral Energy Research (WPI-I2CNER), Kyushu University, 744 Motooka, Nishi-ku, Fukuoka 819-0395, Japan

${ }^{6} J S T$, ERATO Takahara Soft Interfaces Project, Kyushu University, 744 Motooka, Nishi-ku, Fukuoka 819-0395, Japan

${ }^{7}$ Institute of Multidisciplinary Research for Advanced Materials (IMRAM), Tohoku University, 2-1-1 Katahira, Aoba-ku, Sendai 980-8577, Japan

\begin{abstract}
The surface dynamics of polyhedral oligomeric silsesquioxane (POSS)-functionalized polystyrene (PS-POSS) thin films above the glass transition temperature were studied by grazing-incidence X-ray photon correlation spectroscopy in order to elucidate the effects of POSS at the end of the polystyrene chains. Much slower fluctuations were observed in the surface of PS-POSS thin films than in the polystyrene thin films, despite the negligible difference in their bulk viscosity. Quantitative analysis based on fluctuation-dissipation theorem indicated that there is a high-viscosity layer at the surface of the PS-POSS films, and that the substrate interface originated from POSS segregation.
\end{abstract}

\section{Introduction}

The stability of polymer thin films is important in applications such as coatings and dielectric layers. Various methods of inhibiting dewetting of the layers have been developed, such as modifying the substrate, adding a nanofiller, and cross-linking the polymer[1-4]. Adding polyhedral oligomeric silsesquioxanes (POSS) to thin films is an efficient way to prevent them from dewetting. For example, Hosaka et al. stabilized polystyrene (PS) thin films by inhibiting dewetting with dispersed phenethyl-POSS and octacyclopentyl-POSS [5, 6] Miyamoto et al. 
also observed the inhibition of dewetting using octacyclopentyl-POSS-terminated PS thin films (PS-POSS) [7]. Miyamoto et al. found that the low molecular weight PS-POSS $\left(M_{\mathrm{n}} \approx 2000\right)$ produced a large difference in the dewetting behavior, whereas the high molecular weight analog $\left(M_{\mathrm{n}} \approx 40,000\right)$ produced a small difference [7]. They investigated the structures of thin films by neutron reflectivity measurements, and discussed the suppression of dewetting in terms of the segregation of POSS at the surface and at the substrate interface. However, the question of how the segregation of POSS affects the inhibition of dewetting remains unsettled. To answer this fundamental question, the physical properties, such as viscosity and surface tension, of PS-POSS thin films rather than bulk PS-POSS should be examined.

Above the glass transition temperature, thermally agitated capillary waves are generated and damped continually at the surface of polymer thin films. The capillary wave spectra for homogeneous liquids have been studied by fluctuation-dissipation theorem, and they were described by using the viscosity, surface tension, and density in the thin films [8,9]. The dynamics of capillary waves have been experimentally studied by coherent scattering techniques, such as surface dynamic light scattering [10,11] and grazing-incidence X-ray photon correlation spectroscopy (GI-XPCS) [12-17]. Kim et al. performed GI-XPCS for linear PS thin films, and observed the capillary waves as predicted by the hydrodynamic theory for homogeneous thin films with bulk viscosity [18]. The unique properties of the thin films can be determined by the dynamical behavior of capillary waves on the films. Wang et al. measured the surface fluctuations of branched-polymer thin films and found that the viscosities calculated from the capillary wave spectra of thin films were 100 times higher than in the bulk [16]. Jiang et al. considered the dynamics of bilayer as a representative example of inhomogeneous thin films, and calculated the surface and interfacial dynamics of polymeric liquid bilayer films based on fluctuation-dissipation theorem [19].

The dynamics of the capillary waves reflects the physical properties of thin films in thin film conditions, and thus the inhomogeneity of thin films can be discussed by investigating the dynamics of capillary waves. In this study, we investigated the capillary waves on PS and PS-POSS thin films with different molecular weights $\left(M_{\mathrm{n}}=40 \mathrm{k}, 25 \mathrm{k}\right.$, and $\left.2.3 \mathrm{k}\right)$ by using GI-XPCS. For high molecular weight polymers $\left(M_{\mathrm{n}}=40 \mathrm{k}\right.$ and $\left.25 \mathrm{k}\right)$, no major difference was observed between the PS and PS-POSS thin films, and the dynamics of the capillary waves were described by uniform viscosity and their viscosities were same as the bulk viscosity throughout the films. However, for low molecular weight polymers $\left(M_{\mathrm{n}}=2.3 \mathrm{k}\right)$, unique behavior was observed for the PS-POSS thin films. The difference between the PS and PS-POSS films may originate from the non-uniform structure of the PS-POSS thin films. We quantitatively discuss the capillary wave dynamics of PS-POSS thin films based on the fluctuation-dissipation theorem and discuss the physical properties of the films. 


\section{EXPERIMENTAL}

PS and PS-POSS were prepared via living anionic polymerization. Tetrahydrofuran was transferred to a flask and cooled to $-78{ }^{\circ} \mathrm{C}$. After $5 \mathrm{~min}$, sec-BuLi was added until the solution turned yellow. The flask was removed from the cooling bath and allowed to reach room temperature, upon which the solution became colorless. The solution was cooled to $-78{ }^{\circ} \mathrm{C}$ again, and $1.07 \mathrm{M}$ sec-BuLi solution in hexane/cyclohexane was added. Styrene was added, turning the mixture orange. After polymerization, half of the reaction mixture was poured into a large amount of methanol to prepare proton-terminated PS (Fig. 1 (a)). The other half was quenched with $\mathrm{CO}_{2}$ to obtained carbonyl-terminated PS (PS-COOH), and precipitated in a large amount of methanol/HCl. The polymers were filtered and dried under vacuum at $100{ }^{\circ} \mathrm{C}$ for $24 \mathrm{~h}$. PS-POSS (Fig. 11 (b)) was obtained by adding 1-(3amino)propyl-3,5,7,9,11,13,15-isooctylpentacyclo[9.5.1.1 $1^{3,9} \cdot 1^{5,15} \cdot 1^{7,13}$ ]octasiloxane (POSS(isooctyl)) (Hybrid Plastics) and 1,4-dioxane, $N, N^{\prime}$-dicyclohexylcarbodiimide to a 20 wt $\%$ solution of PS-COOH in 1,4-dioxane. The mixture was stirred at $50{ }^{\circ} \mathrm{C}$ for $12 \mathrm{~h}$, filtered, and then poured into a large amount of hexane. The residue was filtered and dried under vacuum at $100{ }^{\circ} \mathrm{C}$ for $24 \mathrm{~h}$. The molecular weight and POSS content of the PS and PS-POSS are summarized in Table 1. The molecular weight of the PS moieties of PS-POSS40k, PS-POSS25k, and PS-POSS2.3k were the same as for PS40k, PS25k, and PS2.3k, respectively, because they were obtained from the same mixture.

We investigated the dynamics of the surface fluctuation of PS and PS-POSS thin films with $M_{\mathrm{n}}=40 \mathrm{k}, 25 \mathrm{k}$, and $2.3 \mathrm{k}$ spin-coated on silicon wafer substrates using cyclopentanone as a solvent. Prior to the film coating, the silicon wafer substrates were cleaned by piranha solution $\left(\mathrm{H}_{2} \mathrm{O}_{2}(30 \%) / \mathrm{H}_{2} \mathrm{SO}_{4}(70 \%)(\mathrm{v} / \mathrm{v})\right)$ for $2 \mathrm{~h}$, and rinsed with deionized water. Before the GI-XPCS measurements the thin films were annealed above the glass transition temperature for $24 \mathrm{~h}$ at $423 \mathrm{~K}$ for molecular weights of $25 \mathrm{k}$ and $40 \mathrm{k}$, and for $12 \mathrm{~h}$ at $383 \mathrm{~K}$ for the molecular weight of $2.3 \mathrm{k}$. No dewetting of thin films was observed after annealing and the GI-XPCS measurements.

The GI-XPCS measurements were performed on the BL19LXU beamline of SPring- 8 with a 27-m-long undulator [20]. The undulator source and $\mathrm{Si}(111)$ monochromator were tuned to an energy of $7.30 \mathrm{keV}$. The incident X-rays with transverse coherence of $16 \times 125 \mu \mathrm{m}(\mathrm{H} \times \mathrm{V})$ at the sample position were cut by slits with dimensions of $20 \times 20 \mu \mathrm{m}$. The parasitic scattering was shielded by the other slits. The experimental geometry is illustrated schematically in Fig. 2. The sample was irradiated with partially coherent X-rays in a vacuum with an incident angle of $0.14^{\circ}$, which was below the critical angle for total external reflection. Direct and specular beam were hidden by beam-stop, and the off-specular diffuse scattering, in which exit angles $\sim 0.25-$ $0.6^{\circ}$, from the polymer surface was recorded. The surface fluctuations in the irradiated area of 
polymer thin films were measured through the scattered X-rays. The scattered X-rays were detected by a two-dimensional hybrid pixel array detector (PILATUS 100K, DECTRIS) with a grid mask resolution enhancer (the effective detected area of each pixel is $\sim 45 \mu \mathrm{m}$ in diameter) mounted $\sim 3.5 \mathrm{~m}$ downstream of the sample [21-23]. For the measurements, 5000-20,000 images were taken with an exposure time of $10-300 \mathrm{~ms}$ and a readout time of $3 \mathrm{~ms}$. During the GI-XPCS measurements, the thin films with $M_{\mathrm{n}}=25 \mathrm{k}$ and $40 \mathrm{k}$ were kept at $413 \mathrm{~K}$, and the thin films with $M_{\mathrm{n}}=2.3 \mathrm{k}$ were kept at $393 \mathrm{~K}$. For investigating the thickness dependence, three different thickness of each polymer were measured: 50, 81, and $146 \mathrm{~nm}$ for PS40k; 50, 84, and $142 \mathrm{~nm}$ for PS-POSS40k; 44, 89, and $155 \mathrm{~nm}$ for PS25k; 44, 79, and $144 \mathrm{~nm}$ for PS-POSS25k; 47, 58, and $76 \mathrm{~nm}$ for PS2.3k; and 35, 52, and $61 \mathrm{~nm}$ for PS-POSS2.3k. The thicknesses were measured by an ellipsometer (MASS-103FH, Five lab.) or interferometer (Optical NanoGauge C11627, Hamamatsu Photonics).

During GI-XPCS measurements, the fluctuation of the scattering intensity, $I(\mathbf{q}, t)$, at a scattering vector $\mathbf{q}$, is obtained in a time series, $t$, and the intensity time-autocorrelation function $g_{2}(\mathbf{q}, t)$ is evaluated as

$$
g_{2}(\mathbf{q}, t)=\left\langle I\left(\mathbf{q}, t^{\prime}\right) I\left(\mathbf{q}, t^{\prime}+t\right)\right\rangle /\left\langle I\left(\mathbf{q}, t^{\prime}\right)\right\rangle^{2},
$$

where the angle brackets indicate time-averaging. In the evaluation, $g_{2}(\mathbf{q}, t)$ is averaged over all pixels within area belonging to $\left|\delta \mathbf{q}_{/ /}\right|=0.5 \times 10^{-3} \mathrm{~nm}^{-1}$ where the vector $\mathbf{q}_{/ /}$indicates the

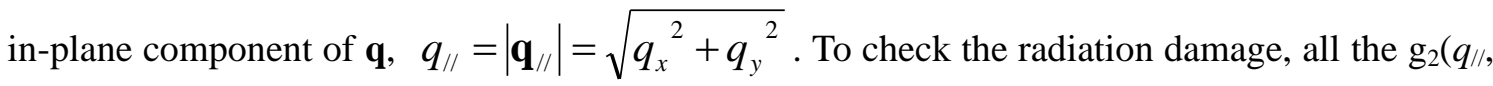
t) for last 1000 frames were compared to that for first 1000 frames. In the present study, the deviations were small enough compared to error bars shown in figures later.

The glass transition temperature, $T_{\mathrm{g}}$, was measured by differential scanning calorimetry with a colorimeter (EXSTRA DSC-6000, Seiko Instruments Inc.). The viscosities, $\eta$, of bulk polymers were measured by using a rotational rheometer (Physica MCR101, Anton Paar). To determine the surface tension, $\gamma$, the contact angles, $\theta$, of four from the 1-alkyl-3-methylimidazolium bis(trifluoromethylsulfonyl)amide $\left[\mathrm{C}_{n} \operatorname{mim}\right]\left[\mathrm{Ntf}_{2}\right](n=2,4,6,10)$ ionic liquids were measured on thin films of PS25k, PS40k, PS-POSS25k, and PS-POSS40k at $413 \mathrm{~K}$ and PS2.3k and PS-POSS2.3k at $393 \mathrm{~K} . \gamma$ was determined by linear extrapolation of the surface tension data of the ionic liquids [24] to $\cos \theta=1$. The measured values of $T_{\mathrm{g}}, \eta$, and $\gamma$ are summarized in Tables 2 and 3.

X-ray photoelectron spectroscopy (XPS) depth profiles (PHI 5000 VersaProbe, ULVAC-PHI) were obtained with an Ar gas cluster ion beam gun (GCIB) with a monochromatic Al-Ka X-ray source (1486.6 eV) [25] [26]. The Ar GCIB gun was operated at a $2.5 \mathrm{kV}$ 
accelerating voltage and a $5 \mathrm{nA}$ beam current. The photoelectron take-off angle was maintained at $45^{\circ}$. High-resolution $\mathrm{C} 1 \mathrm{~s}, \mathrm{Si} 2 \mathrm{p}$ and $\mathrm{O} 1 \mathrm{~s}$ spectra were acquired at $0.1 \mathrm{eV}$ steps for PS-POSS2.3k (44-nm-thick), PS-POSS25k (105-nm-thick) and PS-POSS40k (120-nm-thick) thin films.

Grazing incidence small angle scattering (GI-SAXS) measurements were performed at BL45XU beamline in SPring-8 [27]. The energy of the X-ray beam was $12.40 \mathrm{keV}$, and the scattering profiles were obtained by two dimensional detector PILATUS 3X 2M (DECTRIS) mounted at $2567 \mathrm{~mm}$ downstream of the sample. The thin film samples were irradiated with an incident angle of $0.08^{\circ}$, which was below the critical angle for total external reflection. The geometry was almost same as Fig. 2 and the off-specular diffuse scattering was measured for PS-POSS2.3k (47-nm-thick), PS-POSS25k (78-nm-thick) and PS-POSS40k (47-nm-thick).

\section{Results and Discussion}

\section{A. Static structure of PS-POSS thin films}

To investigate the structure of our PS-POSS thin films, we performed Ar gas cluster sputtering XPS measurements. Figure 3 (a)-(c) show the XPS spectra for Si 2p from the thin films of PS-POSS2.3k, PS-POSS25k and PS-POSS40k sputtered by Ar gas cluster ion beam at sputter time of $0,0.4,0.8,1.2$ and $1.6 \mathrm{~min}$, which corresponds to the depth from the surface of 0 , $1.20,2.39,3.59$ and $4.79 \mathrm{~nm}$. At the surface (sputter time of $0 \mathrm{~min}$ ) the peaks at $\sim 101 \mathrm{eV}$ were observed for all the PS-POSS thin films, and the intensity of the peaks became lower with increasing the sputter time. Figure 3 (d) shows the depth profile of the signal ratio of the XPS spectrum, $I_{\mathrm{si} 2 \mathrm{p}} / I_{\mathrm{C} 1 \mathrm{~s}}$, for PS-POSS2.3k, PS-POSS25k, and PS-POSS40k, where $I_{\mathrm{si} 2 \mathrm{p}}$ and $I_{\mathrm{C} 1 \mathrm{~s}}$ are the integrated area of the Si $2 \mathrm{p}$ peak and C 1 s peak, respectively. $I_{\mathrm{si2p}} / I_{\mathrm{Cls}}$ for all the films at the surfaces are higher than that of inside of the films. Furthermore $I_{\mathrm{si2p}} / I_{\mathrm{Cls}}$ for PS-POSS2.3k near the surface is much higher than that for the other films. These results represent the enrichment of $\mathrm{Si}$ on the surfaces of all films, indicating the segregation of POSS at the surface. Moreover the POSS at the surface of PS-POSS2.3k thin film is more segregated than that of PS-POSS25k and PS-POSS40k thin films.

Regarding the structure of near substrate, the clear evidence of POSS segregation at the substrate was not obtained from the Si $2 p$ spectra since the peaks for POSS could not be distinguished from the peaks for the substrate. Then we checked the spectra for O 1s. Figure 4 (a)-(c) show the XPS spectra for O 1s from the thin films of PS-POSS2.3k, PS-POSS25k and PS-POSS40k at various sputter time.

At the surface, sputter time of $0 \mathrm{~min}(\mathrm{depth}=0 \mathrm{~nm})$, the peak at $\sim 531 \mathrm{eV}$ were observed for all the PS-POSS thin films while the apparent peak was not observed inside the films obtained at sputter time of $4.8 \mathrm{~min}$. These peaks at $\sim 531 \mathrm{eV}$ can be assigned as POSS originating peak 
because they were not observed from a homo-PS thin film (data are not shown) at any sputter time but observed from only PS-POSS thin films. From the substrates, the peaks at $\sim 532.5 \mathrm{eV}$, which originating from $\mathrm{SiO}_{2}$ layer on the silicon substrate, were observed for all the thin films at enough large sputter time. The spectrum for the PS-POSS2.3k thin film at sputter time of 10.6 min, which corresponds to near the substrate, contains two peaks, originating from POSS and originating from the substrate. This result indicates POSS segregation in the thin films near the substrate. On the other hand, for PS-POSS25k and PS-POSS40k thin films, no apparent peak originating from POSS were observed near the substrate. Due to the low contents of POSS in PS-POSS25k and PS-POSS40k, the peak from POSS might be very small and negligible. Figure 4 (d) shows the depth profile of the signal ratio of the XPS spectrum, $I_{\mathrm{O} 1 \mathrm{~s}}{ }^{\mathrm{POSS}} / I_{\mathrm{Cls}}$, for PS-POSS2.3k, where $I_{\mathrm{O} 1 \mathrm{~s}}{ }^{\text {POSS }}$ is the integrated area of the $\mathrm{O} 1 \mathrm{~s}$ peak originating from POSS at $\sim 531 \mathrm{eV}$. This profile clearly shows POSS segregation at the surface and the near substrate.

The inplane structure of the surface of PS-POSS thin films were studied by GI-SAXS measurements. Figure 5 shows the intensity profile against $\mathrm{q}_{/ /}$for PS-POSS2.3k, PS-POSS25k and PS-POSS40k thin films. The profile for PS-POSS2.3k multiplied by 0.5 was also plotted for comparison with the other profiles. These profiles showed similar curves, and no apparent features were observed indicating the inplane structure.

These results agree with the reported results from the neutron reflectivity measurements by Miyamoto et al. [7]. They studied the structure of PS-POSS thin films by the neutron reflectivity measurements of deuterated PS-POSS thin films, and they found that the POSS moieties of dPS-POSS formed an enrichment layer at the surface and the substrate interface. Furthermore, there was a dPS-rich layer between the POSS-rich layer and the center bulk layer.

Based on these results for PS-POSS thin films, schematic drawing of the structure of PS-POSS thin films could be depicted. Figure 9 (a) shows a schematic drawing of the low molecular weight PS-POSS $\left(M_{\mathrm{n}}=2.3 \mathrm{k}\right)$ thin film. In this case PS-POSS thin films were composed of a surface layer, bulk layer, and substrate interface layer. In the bulk layer, the PS-POSS chains were oriented randomly. In contrast, in the surface layer and the substrate interface layer, the PS-POSS chains were oriented perpendicularly, with the POSS part facing the air and the substrate. Figure 9 (b) shows a schematic drawing of the high molecular weight PS-POSS $\left(M_{\mathrm{n}}=25 \mathrm{k}\right.$ and $\left.40 \mathrm{k}\right)$ thin films. The segregation of POSS could be much weaker than that of PS-POSS2.3k thin films and the layer structure could be unclear.

\section{B. Dynamics for high molecular weight PS and PS-POSS thin films ( $M_{\mathrm{n}}=25 \mathrm{k}$ and $\left.40 \mathrm{k}\right)$}

Figure 7 (a) shows representative data for the measured $g_{2}\left(q_{\| /}, t\right)$ of the high molecular weight PS and PS-POSS thin films. They were taken at $q_{/ \prime}=7.74 \times 10^{-3} \mathrm{~nm}^{-1}$ for PS25k and PS-POSS25k with thicknesses of $44 \mathrm{~nm}$ at $413 \mathrm{~K}$. There were no clear differences between 


\section{PS25k and PS-POSS25k.}

The relaxation rates of $g_{2}\left(q_{/ /}, t\right)$ are evaluated by fitting to an exponential function,

$$
g_{2}\left(q_{/ /}, t\right)=\beta \exp [-2 \Gamma t]+1,
$$

where $\beta$ is the speckle contrast and $\Gamma$ is the relaxation rate. Typical values of $\beta$ were $0.01-0.03$ with a slight $q_{/ /}$dependence, which was consistent with the estimated value from the speckle size and the hole size of the grid mask on the detector. Equation (2) fits the experimental $g_{2}\left(q_{/ /,}, t\right)$ data (solid lines, Fig. 7 (a)) with $\Gamma=3.73 \times 10^{-2}$ and $3.63 \times 10^{-2} \mathrm{~s}^{-1}$ for PS25k and PS-POSS25k, respectively. The values of $\Gamma$ for PS25k and PS-POSS25k obtained from the fitting analysis with Eq. (2) are plotted against $q_{/ /}$in Fig. 7 (b) and are similar over the measured $q_{/ /}$range of $0.4<q_{/ /}<1.6 \times 10^{-2} \mathrm{~nm}^{-1}$.

Based on hydrodynamic theory and the fluctuation-dissipation theorem [8, 9], the $q_{/ /}$-dependence of $\Gamma$ in the overdamped regime on the surface of a uniformly viscous liquid polymer thin film with a nonslip boundary condition underneath is

$$
\Gamma=\frac{\gamma}{2 \eta} q_{/ /} \frac{\sinh \left(q_{1 /} d\right) \cosh \left(q_{1 /} d\right)-q_{/ 1} d}{\cosh ^{2}\left(q_{/ /} d\right)+\left(q_{/ /} d\right)^{2}},
$$

where $d$ is the film thickness. The solid lines for PS25k and PS-POSS25k in Fig. 7 (b) are the dispersion curves calculated from Eq. (3a) with the parameters in Table 2. The experimental data showed good agreement with the calculated lines. For various film thicknesses, Eq. (3a) is rewritten as

$$
\Gamma d=\frac{\gamma}{2 \eta}\left(q_{/ /} d\right) \frac{\sinh \left(q_{/ /} d\right) \cosh \left(q_{/ /} d\right)-\left(q_{/ /} d\right)}{\cosh ^{2}\left(q_{/ /} d\right)+\left(q_{/ /} d\right)^{2}} .
$$

In Fig. 7 (c), $\Gamma d$ is plotted against $q_{/ /} d$ for PS25k with thicknesses of 44, 89, and $155 \mathrm{~nm}$, and PS-POSS25k with thicknesses of 44, 79, and $144 \mathrm{~nm}$, and the theoretical lines calculated from Eq. (3b) are shown. All the measured data for PS25k and PS-POSS25k thin films with different thickness were almost linear, and showed good agreement with the calculated lines. Similar results were obtained for the thin films of PS40k and PS-POSS40k. Experimental $\Gamma d$ for PS40k thin films thicknesses of 50, 81, and $146 \mathrm{~nm}$, and PS-POSS40k thin film thicknesses of 50, 84 and $142 \mathrm{~nm}$, are plotted against $q_{/ /} d$ in Fig. 8. The result showed good agreement with the theoretical dispersion relation calculated from Eq. (3) with the parameters in Table 2. The GI-XPCS results for PS25k, PS-POSS25k, PS40k, and PS-POSS40k thin films for the PS thin films and the PS-POSS thin films showed similar surface fluctuations. The fluctuations are expressed by the capillary waves on uniformly viscous liquid polymer thin films.

In the spin-coated PS thin films, existence of remaining solvent were reported, and an enrichment of solvent at the Si/PS interfaces were found even if the thin films were annealed [28]. In that case, the lower viscosity layer should be formed at the Si/PS interfaces and the dynamics of capillary waves should become faster and cannot be expressed by only bulk 
viscosity. However, the present data were expressed by the capillary waves on uniformly viscous liquid polymer thin films. The remaining solvent effects might be negligible because the content of remaining solvent was very low due to the molecular weight of polymers were relatively small and the long annealing time in the present case.

\section{GI-XPCS for low molecular weight PS and PS-POSS thin films $\left(M_{\mathrm{n}}=\mathbf{2 . 3 \mathrm { k }}\right)$}

Figure 9 (a) shows representative data for measured $g_{2}\left(q_{/ /}, t\right)$ of PS2.3k and PS-POSS2.3k thin films. The $g_{2}\left(q_{\|}, t\right)$ values were measured for a 58-nm-thick PS2.5k thin film at $q=4.74 \times 10^{-3}$ $\mathrm{nm}^{-1}$ and a 61-nm-thick PS-POSS2.5k thin film at $q_{/ /}=4.76 \times 10^{-3} \mathrm{~nm}^{-1}$ at $393 \mathrm{~K}$. Unlike the high molecular weight PS and PS-POSS thin films, $g_{2}\left(q_{\| /}, t\right)$ for PS-POSS2.3k showed much slower relaxation $\left(\Gamma=1.99 \times 10^{-2} \mathrm{~s}^{-1}\right.$, obtained by fitting the data with Eq. (2)) than that for PS2.3k $\left(\Gamma=6.20 \times 10^{-1} \mathrm{~s}^{-1}\right)$. The values of $\Gamma$ obtained from the fitting analysis with Eq. (2) are plotted against $q_{/ /}$in Fig. 9 (b). $\Gamma$ for PS-POSS2.3k was much smaller than that for PS2.3k over the entire $q_{/ /}$range of our measurements. The solid lines for PS2.3k and PS-POSS2.3k in Fig. 9 (b) are the dispersion curves calculated from Eq. (3) with the parameters in Table 3. Although the measured $\Gamma$ for PS2.3k showed good agreement with the theoretical line (red solid line), the measured $\Gamma$ for PS-POSS2.3k deviated from the theoretical line (black solid line). In Fig. 9 (c) $\Gamma d$ is plotted against $q_{/ /} d$ for PS2.3k with thicknesses of 47, 58, and $76 \mathrm{~nm}$, and PS-POSS2.3k with thicknesses of 35, 52, and $61 \mathrm{~nm}$. The theoretical lines calculated from Eq. (3b) using the parameters in Table 3 are also shown. Although the measured $\Gamma$ for PS2.3k thin films with various thickness were similar and agreed with the theoretical prediction, measured $\Gamma$ for PS-POSS2.3k were far from the theoretical line. Thus, the observed relaxation behavior for PS-POSS2.3k could not be explained by capillary waves on uniformly viscous liquid polymer thin films.

Based on the study of static structure of low molecular weight PS-POSS thin films, we assumed that the PS-POSS2.3k thin films may not be a uniformly viscous liquid layer, but composed of three different viscous liquid layers. Thus, we explained the measured $q_{\prime \prime}$ dependence of $\Gamma$ for PS-POSS2.3k thin films with thicknesses of 35, 53, and $61 \mathrm{~nm}$ by considering the capillary waves of thin films composed of three different viscous liquid layers.

The $q_{/ /}$dependence of relaxation rate of the capillary waves of liquid surface can be derived from the poles of dynamic susceptibility, which specify the linear response of the surface height to a periodic external force field acting vertically on the liquid surface, by using the classical fluctuation-dissipation theorem. The explicit expression of the dynamic susceptibility for the capillary waves of three layer thin films was derived by extending the bilayer model reported by Jiang et al. [19] and is given in the Appendix.

Figure 10 shows a three-layer model on a solid substrate where the bottom, middle, and top 
layers are $h_{1}, h_{2}$, and $h_{3}\left(h_{3}=d-h_{1}-h_{2}\right)$, respectively. Hereafter, we use subscript and superscript 1, 2 and 3 to denote the bottom (I), center (II), and top layers (III), respectively. For example, the viscosity and the mass density of the bottom layer are denoted $\eta_{1}$ and $\rho_{1}$. The interfacial tension between layers I and II, between II and III, and the surface tension are $\gamma_{1}, \gamma_{2}$, and $\gamma_{3}$, respectively. For simplicity, the following assumptions were made: (i) $\eta_{2}$ is same as the bulk viscosity of $1.10 \times 10^{3} \mathrm{~Pa} \cdot \mathrm{s}$; (ii) because the contents of POSS is the same order as shown in Fig. 4 (d), the viscosity of the top layer and the bottom layer are the same $\left(\eta_{1}=\eta_{3}\right)$; (iii) the difference in the density between the layers is negligible and the densities are same as bulk PS $\left(\rho_{1}=\rho_{2}=\rho_{3}=1.0 \mathrm{~g} \cdot \mathrm{cm}^{-3}\right)$; (iv) because the components of layers are the same materials, $\gamma_{1}=\gamma_{2}=0$; and (v) $h_{1}$ and $h_{3}$ are independent from the total thickness, $d$, and are fixed.

Applying the three-layer model with the above assumptions to the measured relaxation rates for PS-POSS thin films with thicknesses of 35,53 , and $61 \mathrm{~nm}$ produced a set of parameters, $\eta_{1}$ ( $\left.=\eta_{3}=9.6 \times 10^{4} \mathrm{~Pa} \cdot \mathrm{s}\right), h_{1}(16 \mathrm{~nm})$, and $h_{3}(17 \mathrm{~nm})$, which describe the $q_{/ /}$dependence of $\Gamma$ with the minimum residual sum of squares. Figure 11 shows the calculated relaxation rates from the three-layer model with these parameters. They agreed well with the measured data for all three thin films with different thicknesses. The viscosities of the surface and substrate interface, $\eta_{1}$ and $\eta_{3}$, were much larger than those in the bulk, meaning that there was a higher viscosity layer at the surface and the substrate interface. This indicates that the molecular motion of PS-POSS was reduced at the top and the bottom layer. Because the dewetting phenomena has been known to have deep correlation with surface fluctuations [29-32], the reduced molecular motion in those layer consequently retarded dewetting behavior, furthermore inhibits the dewetting of PS-POSS thin films in a realistic time window.

The considerable difference of surface dynamics were observed between PS2.3k and PS-POSS2.3k thin films although no major difference was observed between PS and PS-POSS thin films. The origin of these difference should be the large difference of contents, which induced the difference of structure. In the low molecular weight PS-POSS thin films, the layers at surface and substrate interface induced by POSS segregation were relatively robust and strongly affect the surface dynamics. On the other hand, in the high molecular weight PS-POSS thin films, the layers formed by segregation of POSS were weak and thin, thus their effects were weak and negligible.

We will still remain the open question about the deviation of $g_{2}\left(q_{/ /}, t\right)$ from a single exponential function shown in Fig. 7 (a) and Fig. 9 (a). Although the deviation from a single exponential function of $g_{2}\left(q_{\|}, t\right)$ for polymer thin films has been reported near $T_{\mathrm{g}}$ and high molecular weight, the mechanism has not been clarified yet $[33,34]$. In the present study, the deviation from a single exponential function were observed for PS-POSS2.3k while that was not observed for PS2.3k thin films. This may indicate that the non-uniform structure of thin films 
may be the key to solve the question of shape of $g_{2}\left(q_{/ /}, t\right)$.

\section{Conclusion}

To investigate the origin of the inhibition of dewetting by POSS functionalization of PS in thin films, we investigated the dynamical behavior of the capillary waves on PS and PS-POSS thin films for molecular weights of $40 \mathrm{k}, 25 \mathrm{k}$, and $2.3 \mathrm{k}$ by GI-XPCS. There was no difference in the dynamics of the capillary waves between the PS and PS-POSS thin films for molecular weights of $40 \mathrm{k}$ and $25 \mathrm{k}$. In contrast, for a molecular weight of $2.3 \mathrm{k}$, the dynamics of the PS-POSS thin films were much slower than those of PS thin films. Although the dynamics of the capillary waves of the PS-POSS2.3k thin films were not explained by the uniformly viscous thin film model, they were explained by considering the presence of high-viscosity layers at the surface and the substrate interface.

\section{Acknowledgement}

The authors thank the staff of BL19LXU and BL45XU at SPring-8 for technical support, and S. Narayanan for advice on the GI-XPCS experiments. The GI-XPCS experiments at BL19LXU were performed under the approval of RIKEN (Proposal No. 20130065, 20140079, and 20150069). The GI-SAXS experiments at BL45XU were performed under the approval of RIKEN (Proposal No. 20160057). This work was partially supported by the ImPACT Program of Council for Science, Technology and Innovation (Cabinet Office, Government of Japan). TH acknowledges JSPS Grant-in-Aid for Young Scientists (B) (25790082) and the JST, PRESTO, for funding the project "Molecular technology and creation of new functions." 


\section{Appendix: Capillary wave spectra of polymeric three-layer films}

The capillary wave spectra of the thermally excited capillary waves on homogeneous supported liquid single-layer films [9] and bilayer films [19] have been studied theoretically. Extending the bilayer model by Jiang et al., the capillary wave spectra of three-layer films and the wave vector dependence of the relaxation rate are derived here.

On a liquid three-layer film on a solid substrate (Fig. 7), we assume small periodic externally applied pressure perturbations on the interface and the free surface of the form $P_{z, 0}^{(1)} e^{i(k x-\omega t)}$, $P_{z, 0}^{(2)} e^{i(k x-\omega t)}$ and $P_{z, 0}^{(3)} e^{i(k x-\omega t)}$, with $u_{z}^{(1)}, \boldsymbol{u}_{z}^{(2)}$ and $\boldsymbol{u}_{z}^{(3)}$ being the vertical displacements of the reactive fluctuations.

Incompressible liquid is governed by the linearized Navier-Stokes equation

$$
\partial_{t} \mathbf{v}=-\frac{1}{\rho} \nabla p+v \nabla^{2} \mathbf{v}
$$

and

$$
\operatorname{div} \mathbf{v}=0
$$

where $\mathbf{v}, p$ and $v=\eta / \rho$ are velocity, pressure, and kinematic viscosity of the liquid, respectively. The solutions of the velocity and pressure in the form of monochromatic plane waves along the $x$ direction are considered,

$$
\begin{gathered}
\mathbf{v}^{(j)}(\mathbf{r}, t)=\left[V_{x}^{(j)}(z) \mathbf{e}_{x}+V_{z}^{(j)}(z) \mathbf{e}_{z}\right] e^{i(k x-\omega t)}, \\
\boldsymbol{p}^{(j)}(\mathbf{r}, t)=\boldsymbol{P}^{(j)}(z) \boldsymbol{e}^{i(k x-\omega t)},
\end{gathered}
$$

where the superscript $j=1,2$, and 3 denotes the solutions in regions I, II, and III, respectively. Substituting Eqs. (A3a) into (A2), we obtain the expression for velocity amplitudes of

$$
i k V_{x}^{(j)}+\partial_{z} V_{z}^{(j)}=0 .
$$

Eq. (A1) leads to the preliminary solutions of the Navier-Stokes equation in regions I, II, and III of

$$
\begin{gathered}
V_{z}^{(j)}(z)=A_{k}^{(j)} \cosh (k z)+B_{k}^{(j)} \sinh (k z)+i k \hat{B}_{j} \cosh \left(\kappa_{j} z\right)+i k \hat{C}_{j} \sinh \left(\kappa_{j} z\right) \\
V_{x}^{(j)}(z)=\frac{i}{k} \partial_{z} V_{z}^{(j)}(z) \\
\frac{P^{(j)}(z)}{\rho_{j}}=\frac{i v_{j}}{k}\left(\kappa_{j}^{2}-\partial_{z}^{2}\right) V_{x}^{(j)}(z)
\end{gathered}
$$


where $\kappa_{j}=\sqrt{k^{2}-i \omega / v_{j}}$, and $A_{k}^{(j)}, B_{k}^{(j)}, \hat{B}_{j}$, and $\hat{C}_{j}$ are constants to be determined by the boundary conditions at $z=0, h_{1}, h_{1}+h_{2}$, and $d$.

\section{(i) Nonslip boundary condition at $z=0$}

The nonslip boundary condition at the substrate interface, $\left.V_{x}^{(1)}\right|_{z=0}=\left.V_{z}^{(1)}\right|_{z=0}=0$, gives

$$
A_{k}^{(1)}=-i k \hat{B}_{k}^{(1)}, \quad B_{k}^{(1)}=-i \kappa_{1} \hat{C}_{1} .
$$

The $x z$ and $z z$ components of the stress tensor in regions I, II, and III are denoted as

$$
\begin{gathered}
\sigma_{x z}^{(j)} \equiv \hat{\sigma}_{x z}^{(j)} e^{i(k x-\omega t)}=\eta_{j}\left(\partial_{z} v_{x}^{(j)}+\partial_{x} v_{z}^{(j)}\right), \\
\sigma_{z z}^{(j)} \equiv \hat{\sigma}_{z z}^{(j)} e^{i(k x-\omega t)}=-p^{(j)}+2 \eta_{j} \partial_{z} v_{z}^{(j)}
\end{gathered}
$$

Then Eqs. (A5), (A6), and (A7a) can be combined into compact matrix expressions as

$$
\begin{aligned}
& \mathbf{X}=\mathbf{B Z}, \text { region I, } \\
& \mathbf{W}=\mathbf{A Y}, \text { region II, } \\
& \mathbf{R}=\mathbf{L S}, \text { region III, }
\end{aligned}
$$

where $\mathbf{X}$ and $\mathbf{Z}, \mathbf{W}$, and $\mathbf{Y}, \mathbf{R}$ and $\mathbf{L}$ are defined for regions I, II, and III, respectively, as

$$
\begin{gathered}
\mathbf{X}=\left[\begin{array}{c}
V_{x}^{(1)} \\
V_{z}^{(1)} \\
P^{(1)} / \rho_{1} \\
\hat{\sigma}_{x z}^{(1)}
\end{array}\right], \mathbf{Z}=\left[\begin{array}{c}
\hat{B}_{1} \\
\hat{C}_{1}
\end{array}\right], \\
\mathbf{W}=\left[\begin{array}{c}
V_{x}^{(2)} \\
V_{z}^{(2)} \\
P^{(2)} / \rho_{2} \\
\hat{\sigma}_{x z}^{(2)}
\end{array}\right], \quad \mathbf{Y}=\left[\begin{array}{c}
A_{k}^{(2)} \\
B_{k}^{(2)} \\
\hat{B}_{2} \\
\hat{C}_{2}
\end{array}\right], \\
\mathbf{R}=\left[\begin{array}{c}
V_{x}^{(3)} \\
V_{z}^{(3)} \\
P^{(3)} / \rho_{3} \\
\hat{\sigma}_{x z}^{(3)}
\end{array}\right], \mathbf{S}=\left[\begin{array}{c}
A_{k}^{(3)} \\
B_{k}^{(3)} \\
\hat{B}_{3} \\
\hat{C}_{3}
\end{array}\right],
\end{gathered}
$$

and the matrices $\mathbf{B}, \mathbf{A}$, and $\mathbf{L}$ are defined as 
$\mathbf{B}=\left[\begin{array}{cc}k \sinh (k z)-\kappa_{1} \sinh \left(\kappa_{1} z\right) & \kappa_{1} \cosh (k z)-\kappa_{1} \cosh \left(\kappa_{1} z\right) \\ -i k \cosh (k z)+i k \cosh \left(\kappa_{1} z\right) & -i \kappa_{1} \sinh (k z)+i k \sinh \left(\kappa_{1} z\right) \\ \omega \sinh (k z) & \omega\left(\kappa_{1} / k\right) \cosh (k z) \\ 2 \eta_{1} k^{2} \cosh (k z)-\eta_{1}\left(k^{2}+\kappa_{1}^{2}\right) \cosh \left(\kappa_{1} z\right) & 2 \eta_{1} k \kappa_{1} \sinh (k z)-\eta_{1}\left(k^{2}+\kappa_{1}^{2}\right) \sinh \left(\kappa_{1} z\right)\end{array}\right]$

(A10a)

$$
\mathbf{A}=\left[\begin{array}{cccc}
i \sinh (k z) & i \cosh (k z) & -\kappa_{2} \sinh \left(\kappa_{2} z\right) & -\kappa_{2} \cosh \left(\kappa_{2} z\right) \\
\cosh (k z) & \sinh (k z) & i k \cosh \left(\kappa_{2} z\right) & i k \sinh \left(\kappa_{2} z\right) \\
(i \omega / k) \sinh (k z) & (i \omega / k) \cosh (k z) & 0 & 0 \\
2 i k \eta_{2} \cosh (k z) & 2 i k \eta_{2} \sinh (k z) & -\eta_{2}\left(k^{2}+\kappa_{2}^{2}\right) \cosh \left(\kappa_{2} z\right) & -\eta_{2}\left(k^{2}+\kappa_{2}^{2}\right) \sinh \left(\kappa_{2} z\right)
\end{array}\right]
$$

, (A10b)

$$
\mathbf{L}=\left[\begin{array}{cccc}
i \sinh (k z) & i \cosh (k z) & -\kappa_{3} \sinh \left(\kappa_{3} z\right) & -\kappa_{3} \cosh \left(\kappa_{3} z\right) \\
\cosh (k z) & \sinh (k z) & i k \cosh \left(\kappa_{3} z\right) & i k \sinh \left(\kappa_{3} z\right) \\
(i \omega / k) \sinh (k z) & (i \omega / k) \cosh (k z) & 0 & 0 \\
2 i k \eta_{3} \cosh (k z) & 2 i k \eta_{3} \sinh (k z) & -\eta_{3}\left(k^{2}+\kappa_{3}^{2}\right) \cosh \left(\kappa_{3} z\right) & -\eta_{3}\left(k^{2}+\kappa_{3}^{2}\right) \sinh \left(\kappa_{3} z\right)
\end{array}\right]
$$

(A10c)

The hydrodynamic problem reduces to the problem of solving for coefficients $\mathbf{Z}, \mathbf{Y}$, and $\mathbf{S}$ via the three boundary conditions at $z=h_{1}, h_{1}+h_{2}$, and $d$.

\section{ii) Interfacial boundary condition at $z=h_{1}$}

The boundary condition of the equal velocities of the liquid on both sides at the interface gives

$$
V_{x}^{(2)}=V_{x}^{(1)}, V_{z}^{(2)}=V_{z}^{(1)},
$$

and the equilibrium of force at the interface gives

$$
\begin{gathered}
\hat{\sigma}_{x z}^{(2)}=\hat{\sigma}_{x z}^{(1)}, \\
\hat{\sigma}_{z z}^{(2)}-\hat{\sigma}_{z z}^{(1)}=\left[\gamma_{1} k^{2}+g\left(\rho_{1}-\rho_{2}\right)\right] U_{z}^{(1)}-P_{z, 0}^{(1)},
\end{gathered}
$$

where $U_{z}^{(1)}$ is the amplitude of the vertical displacement of interfacial fluctuations expressed by $u_{z}^{(1)}=U_{z}^{(1)} e^{i(k x-\omega t)}$. Using Eqs. (A4) and (A7c) and relation $u_{z}^{(1)}=\left.\frac{i}{\omega} v_{z}^{(1)}\right|_{z=h_{1}}$, derived from $\left.v_{z}^{(1)}\right|_{z=h_{1}}=\partial_{t} u_{z}^{(1)}$, Eqs. (A11) can be rewritten as 


$$
\left.\mathbf{W}\right|_{z=h_{1}}=\left.\mathbf{T X}\right|_{z=h_{1}}+\mathbf{U},
$$

where

$$
\begin{gathered}
\mathbf{U}=\left[\begin{array}{c}
0 \\
0 \\
P_{z, 0}^{(1)} / \rho_{2} \\
0
\end{array}\right], \\
\mathbf{T}=\left[\begin{array}{cccc}
1 & 0 & 0 & 0 \\
0 & 1 & 0 & 0 \\
\frac{2 i k\left(\eta_{1}-\eta_{2}\right)}{\rho_{2}} & -\frac{i\left(\gamma_{1} k^{2}+g\left(\rho_{1}-\rho_{2}\right)\right)}{\rho_{2} \omega} & \frac{\rho_{1}}{\rho_{2}} & 0 \\
0 & 0 & 0 & 1
\end{array}\right] .
\end{gathered}
$$

Combining Eqs. (A8) and (A12), $\mathbf{Y}$ (for region II) and $\mathbf{Z}$ (for region I) can be related as

$$
\begin{gathered}
\mathbf{Y}=\left.\mathbf{A}^{-1} \mathbf{W}\right|_{z=h_{1}}=\left.\mathbf{A}^{-1} \mathbf{T X}\right|_{z=h_{1}}+\left.\mathbf{A}^{-1} \mathbf{U}\right|_{z=h_{1}}=\left.\mathbf{A}^{-1} \mathbf{T B Z}\right|_{z=h_{1}}+\left.\mathbf{A}^{-1}\left[\begin{array}{c}
0 \\
0 \\
P_{z, 0}^{(1)} / \rho_{2} \\
0
\end{array}\right]\right|_{z=h_{1},}, \\
Y_{k}=\sum_{l=1}^{2} C_{k l} Z_{l}+\left.\frac{P_{z, 0}^{(1)}}{\rho_{2}} A_{k 3}^{-1}\right|_{z=h_{1}},
\end{gathered}
$$

where the intermediate matrix $\mathbf{C}$ is given by

$$
\mathbf{C}=\left.\left(\mathbf{A}^{-1} \mathbf{T B}\right)\right|_{z=h_{1}} .
$$

ii) Interfacial boundary condition at $z=h_{1}+h_{2}$

The boundary condition at $z=h_{1}+h_{2}$ are given in the same manner as $z=h_{1}$. The following equations are obtained at $z=h_{1}+h_{2}$,

$$
\begin{gathered}
V_{x}^{(3)}=V_{x}^{(2)}, V_{z}^{(3)}=V_{z}^{(2)}, \\
\hat{\sigma}_{x z}^{(3)}=\hat{\sigma}_{x z}^{(2)}, \\
\hat{\sigma}_{z z}^{(3)}-\hat{\sigma}_{x z}^{(2)}=\left[\gamma_{2} k^{2}+g\left(\rho_{2}-\rho_{3}\right)\right] U_{z}^{(2)}-P_{z, 0}^{(2)} .
\end{gathered}
$$

Eqs. (A15a)-(A15c) are summarized as

$$
\left.\mathbf{R}\right|_{z=h_{1}+h_{2}}=\left.\mathbf{K W}\right|_{z=h_{1}+h_{2}}+\mathbf{J},
$$


where

$$
\begin{gathered}
\mathbf{J}=\left[\begin{array}{c}
0 \\
0 \\
P_{z, 0}^{(2)} / \rho_{3} \\
0
\end{array}\right], \\
\mathbf{K}=\left[\begin{array}{cccc}
1 & 0 & 0 & 0 \\
\frac{2 i k\left(\eta_{2}-\eta_{3}\right)}{\rho_{3}} & -\frac{i\left(\gamma_{2} k^{2}+g\left(\rho_{2}-\rho_{3}\right)\right)}{\rho_{3} \omega} & 0 & 0 \\
0 & 0 & \rho_{2} & 0 \\
\rho_{3} & 0 & 1
\end{array}\right] .
\end{gathered}
$$

Combining Eqs. (A8) and (A16), $\mathbf{S}$ (for region III) and $\mathbf{Y}$ (for region II) can be related,

$\mathbf{S}=\left.\mathbf{L}^{-1} \mathbf{R}\right|_{z=h_{1}+h_{2}}=\left.\mathbf{L}^{-1} \mathbf{K W}\right|_{z=h_{1}+h_{2}}+\left.\mathbf{L}^{-1} \mathbf{J}\right|_{z=h_{1}+h_{2}}=\left.\mathbf{L}^{-1} \mathbf{K A Y}\right|_{z=h_{1}+h_{2}}+\left.\mathbf{L}^{-1}\left[\begin{array}{c}0 \\ 0 \\ P_{z, 0}^{(2)} / \rho_{3} \\ 0\end{array}\right]\right|_{z=h_{1}+h_{2}}$ (A17a)

$$
S_{k}=\sum_{l=1}^{2} F_{k l} Y_{l}+\left.\frac{P_{z, 0}^{(2)}}{\rho_{3}} L_{k 3}^{-1}\right|_{z=h_{1}+h_{2}},
$$

where

$$
\mathbf{F}=\left.\left(\mathbf{L}^{-1} \mathbf{K A}\right)\right|_{z=h_{1}+h_{2}} .
$$

iv) Surface boundary condition at $z=d$

The boundary condition at the free surface gives

$$
\begin{gathered}
\hat{\sigma}_{x z}^{(3)}=\eta_{3}\left(\partial_{z} V_{x}^{(3)}+i k V_{z}^{(3)}\right)=0 \\
\hat{\sigma}_{z z}^{(3)}=-P^{(3)}+2 \eta_{3} \partial_{z} V_{z}^{(3)}=-\left(\gamma_{3} k^{2}+g \rho_{3}\right) U_{z}^{(3)}+P_{z, 0}^{(3)} .
\end{gathered}
$$

From the equation of continuity Eq. (A4), Eq. (A18a) can be rewritten as

$$
\mathbf{D S}=0 \text {, }
$$

where 


$$
\mathbf{D}=\left[\begin{array}{c}
2 k \cosh (k d) \\
2 k \sinh (k d) \\
i\left(k^{2}+\kappa_{3}^{2}\right) \cosh \left(\kappa_{3} d\right) \\
i\left(k^{2}+\kappa_{3}^{2}\right) \sinh (k d)
\end{array}\right]^{\mathrm{T}}
$$

Applying the vertical surface displacement $u_{z}^{(3)}=\left.\frac{i}{\omega} v_{z}^{(3)}\right|_{z=d}$ to Eq. (A18b) gives the expression

$$
\mathbf{E S}=\frac{P_{z, 0}^{(3)}}{\rho_{3}}
$$

where

$$
\mathbf{E}=\left[\begin{array}{l}
\left(2 v_{3} k-\frac{i \omega}{k}\right) \sinh (k d)+\frac{i\left(\gamma_{3} k^{2}+g \rho_{3}\right)}{\rho_{3} \omega} \cosh (k d) \\
\left(2 v_{3} k-\frac{i \omega}{k}\right) \cosh (k d)+\frac{i\left(\gamma_{3} k^{2}+g \rho_{3}\right)}{\rho_{3} \omega} \sinh (k d) \\
2 i v_{3} k \kappa_{3} \sinh \left(\kappa_{3} d\right)-\frac{k\left(\gamma_{3} k^{2}+g \rho_{3}\right)}{\rho_{3} \omega} \cosh \left(\kappa_{3} d\right) \\
2 i v_{3} k \kappa_{3} \cosh \left(\kappa_{3} d\right)-\frac{k\left(\gamma_{3} k^{2}+g \rho_{3}\right)}{\rho_{3} \omega} \sinh \left(\kappa_{3} d\right)
\end{array}\right]^{\mathrm{T}}
$$

Combining Eqs. (A19a), (A14), and (A17) gives the expressions

$\mathbf{D S}=\mathbf{D}\left(\mathbf{F} \mathbf{Y}+\left.\mathbf{L}^{-1}\left[\begin{array}{c}0 \\ 0 \\ P_{z, 0}^{(2)} / \rho_{3} \\ 0\end{array}\right]\right|_{z=h_{1}+h_{2}}\right)=\mathbf{D}\left(\mathbf{F}\left(\mathbf{C Z}+\left.\mathbf{A}^{-1}\left[\begin{array}{c}0 \\ 0 \\ P_{z, 0}^{(1)} / \rho_{2} \\ 0\end{array}\right]\right|_{z=h_{1}}\right)+\left.\mathbf{D L}^{-1}\left[\begin{array}{c}0 \\ 0 \\ P_{z, 0}^{(2)} / \rho_{3} \\ 0\end{array}\right]\right|_{z=h_{1}+h_{2}=0}\right.$

, (A21a)

$$
\sum_{l=1}^{2} P_{l} Z_{l}=-\frac{P_{z, 0}^{(1)}}{\rho_{2}} P_{0}^{h_{1}}-\frac{P_{z, 0}^{(2)}}{\rho_{3}} P_{0}^{h_{1}+h_{2}}
$$

where

$$
P_{l}=(\mathbf{D F C})_{l}, P_{0}^{h_{1}}=\left.\left(\mathbf{D F A}^{-1}\right)_{3}\right|_{z=h_{1}}, P_{0}^{h_{1}+h_{2}}=\left.\left(\mathbf{D L}^{-1}\right)_{3}\right|_{z=h_{1}+h_{2}}
$$

Combining Eqs. (A19b), (A14), and (A17) gives the expressions 
$\mathbf{E S}=\mathbf{E}\left(\mathbf{F Y}+\left.\mathbf{L}^{-1}\left[\begin{array}{c}0 \\ 0 \\ P_{z, 0}^{(2)} / \rho_{3} \\ 0\end{array}\right]\right|_{z=h_{1}+h_{2}}\right)=\mathbf{E}\left(\mathbf{F}\left(\mathbf{C Z}+\left.\mathbf{A}^{-1}\left[\begin{array}{c}0 \\ 0 \\ P_{z, 0}^{(1)} / \rho_{2} \\ 0\end{array}\right]\right|_{z=h_{1}}\right)\right)+\left.L^{-1}\left[\begin{array}{c}0 \\ 0 \\ P_{z, 0}^{(2)} / \rho_{3} \\ 0\end{array}\right]\right|_{z=h_{1}+h_{2}}=\frac{P_{z, 0}^{(3)}}{\rho_{3}}$ , (A22a)

$$
\sum_{l=1}^{2} Q_{l} Z_{l}=-\frac{P_{z, 0}^{(3)}}{\rho_{2}} Q_{0}^{h_{1}}-\frac{P_{z, 0}^{(2)}}{\rho_{3}} Q_{0}^{h_{1}+h_{2}}
$$

where

$$
Q_{l}=(\mathbf{E F C})_{l}, Q_{0}^{h_{1}}=\left.\left(\mathbf{E F A}^{-1}\right)_{3}\right|_{z=h_{1}}, Q_{0}^{h_{1}+h_{2}}=\left.\left(\mathbf{E L}^{-1}\right)_{3}\right|_{z=h_{1}+h_{2}} .
$$

\section{v) Surface and interfacial dynamic susceptibilities}

Solving the Eqs. (A21b) and (A22b) for $\mathbf{Z}$ gives the expressions

$$
\begin{gathered}
Z_{1}=\frac{\left(P_{2} Q_{0}^{h_{1}}-P_{0}^{h_{1}} Q_{2}\right)\left(P_{z, 0}^{(1)} / \rho_{2}\right)+\left(P_{2} Q_{0}^{h_{1}+h_{2}}-P_{0}^{h_{1}+h_{2}} Q_{2}\right)\left(P_{z, 0}^{(2)} / \rho_{3}\right)-P_{2}\left(P_{z, 0}^{(3)} / \rho_{3}\right)}{P_{1} Q_{2}-P_{2} Q_{1}}, \text { (A23a) } \\
Z_{2}=\frac{\left(P_{0}^{h_{1}} Q_{1}-P_{1} Q_{0}^{h_{1}}\right)\left(P_{z, 0}^{(1)} / \rho_{2}\right)+\left(P_{0}^{h_{1}+h_{2}} Q_{1}-P_{1} Q_{0}^{h_{1}+h_{2}}\right)\left(P_{z, 0}^{(2)} / \rho_{3}\right)-P_{1}\left(P_{z, 0}^{(3)} / \rho_{3}\right)}{P_{1} Q_{2}-P_{2} Q_{1}} . \quad \text { (A23b) }
\end{gathered}
$$

Then, the monochromatic plane-wave solutions can be obtained. The amplitudes of vertical interfacial fluctuation between region I and II is given by

$$
\begin{aligned}
U_{z}^{(1)} & =\left.\frac{i}{\omega} V_{z}^{(1)}\right|_{z=h_{1}}=\frac{i}{\omega} \sum_{k=1}^{2} N_{k} Z_{k} \\
& =\frac{i}{\omega} \frac{1}{[\mathbf{P}, \mathbf{Q}]}\left[\frac{P_{0}^{h_{1}}[\mathbf{Q}, \mathbf{N}]-Q_{0}^{h_{1}}[\mathbf{P}, \mathbf{N}]}{\rho_{2}} P_{z, 0}^{(1)}+\frac{[\mathbf{P}, \mathbf{N}]}{\rho_{2}} P_{z, 0}^{(2)}\right],
\end{aligned}
$$

where

$$
\begin{gathered}
{[\mathbf{P}, \mathbf{Q}] \equiv P_{1} Q_{2}-P_{2} Q_{1},} \\
\mathbf{N}=\left[\begin{array}{c}
-i\left[k \cosh \left(k h_{1}\right)-k \cosh \left(\kappa_{1} h_{1}\right)\right] \\
-i\left[\kappa_{1} \sinh \left(k h_{1}\right)-k \sinh \left(\kappa_{1} h_{1}\right)\right]
\end{array}\right]^{\mathrm{T}} .
\end{gathered}
$$

Referring to Eqs. (A14), the amplitudes of vertical interfacial fluctuation between regions II and III is given as 


$$
\begin{aligned}
& U_{z}^{(2)}=\left.\frac{i}{\omega} V_{z}^{(2)}\right|_{z=h_{1}+h_{2}}=\frac{i}{\omega} \sum_{k=1}^{4} G_{k} Y_{k} \\
& =\frac{i}{\omega} \frac{1}{[\mathbf{P}, \mathbf{Q}]}\left[\frac{[\mathbf{M}, \mathbf{P}, \mathbf{Q}]}{\rho_{2}} P_{z, 0}^{(1)}+\frac{\left(P_{2} Q_{0}^{h_{1}+h_{2}}-P_{0}^{h_{1}+h_{2}} Q_{2}\right) M_{1}+\left(P_{0}^{h_{1}+h_{2}} Q_{1}-P_{1} Q_{0}^{h_{1}+h_{2}}\right) M_{2}}{\rho_{3}} P_{z, 0}^{(2)}-\frac{[\mathbf{P}, \mathbf{M}]}{\rho_{3}} P_{z, 0}^{(3)}\right]
\end{aligned}
$$

where

$$
[\mathbf{M}, \mathbf{P}, \mathbf{Q}]=\sum_{i, j, k=0}^{2} \varepsilon_{i j k} M_{i} P_{j} Q_{k},
$$

$\varepsilon_{i j k}$ is the Levi-Cività symbol, and

$$
\mathbf{G}=\left[\begin{array}{c}
\cosh \left(k\left(h_{1}+h_{2}\right)\right) \\
\sinh \left(k\left(h_{1}+h_{2}\right)\right) \\
i k \cosh \left(\kappa_{2}\left(h_{1}+h_{2}\right)\right) \\
i k \sinh \left(\kappa_{2}\left(h_{1}+h_{2}\right)\right)
\end{array}\right]^{\mathrm{T}} .
$$

Referring to Eqs. (A17), the amplitudes of vertical surface fluctuation is given as

$$
\begin{gathered}
U_{z}^{(3)}=\left.\frac{i}{\omega} V_{z}^{(3)}\right|_{z=d}=\frac{i}{\omega} \sum_{k=1}^{4} H_{k} S_{k} \\
=\frac{i}{\omega} \frac{1}{[\mathbf{P}, \mathbf{Q}]}\left[\left\{\Theta_{1}\left(P_{2} Q_{0}^{h_{1}}-P_{0}^{h_{1}} Q_{2}\right)+\Theta_{2}\left(P_{0}^{h_{1}} Q_{1}-P_{1} Q_{0}^{h_{1}}\right)+\Lambda_{3}[\mathbf{P}, \mathbf{Q}]\right\} \frac{P_{z, 0}^{(1)}}{\rho_{2}}\right. \\
+\left\{\Theta_{1}\left(P_{2} Q_{0}^{h_{1}+h_{2}}-P_{0}^{h_{1}+h_{2}} Q_{2}\right)+\Theta_{2}\left(P_{0}^{h_{1}+h_{2}} Q_{1}-P_{1} Q_{0}^{h_{1}+h_{2}}\right)+\Omega_{3}[\mathbf{P}, \mathbf{Q}]\right\} \frac{P_{z, 0}^{(2)}}{\rho_{3}} \\
\left.+\left\{-\Theta_{1} P_{2}+\Theta_{2} P_{1}\right\} \frac{P_{z, 0}^{(3)}}{\rho_{3}}\right] \\
\mathbf{H}=\left[\begin{array}{c}
\cosh (k d) \\
\sinh (k d) \\
i k \cosh \left(\kappa_{3} d\right) \\
i k \sinh \left(\kappa_{3} d\right)
\end{array}\right]^{\mathrm{T}}
\end{gathered}
$$




$$
\mathbf{S}=\mathbf{F} \mathbf{C Z}+\left.\mathbf{F A}^{-1}\left[\begin{array}{c}
0 \\
0 \\
P_{z, 0}^{(1)} / \rho_{2} \\
0
\end{array}\right]\right|_{z=h_{1}}+\left.\mathbf{L}^{-1}\left[\begin{array}{c}
0 \\
0 \\
P_{z, 0}^{(2)} / \rho_{3} \\
0
\end{array}\right]\right|_{z=h_{1}+h_{2}}
$$

Eqs. (A24a), (A25a), and (A26a) can be rewritten as $U_{z}^{(i)}=\sum_{j} \chi_{z z}^{(i j)} P_{z, 0}^{(j)}$. Thus, the self- and cross-dynamic susceptibilities for the interface and the free surface are obtained via the linear response theory are

$$
\begin{gathered}
\chi_{z z}=\frac{i}{\omega} \frac{1}{[\mathbf{P}, \mathbf{Q}]} \mathbf{\Xi} \\
\Xi_{11}=\frac{P_{0}^{h_{1}}[\mathbf{Q}, \mathbf{N}]-Q_{0}^{h_{1}}[\mathbf{P}, \mathbf{N}]}{\rho_{2}}, \Xi_{12}=\frac{[\mathbf{P}, \mathbf{N}]}{\rho_{2}}, \Xi_{13}=\mathbf{O} \\
\Xi_{21}=\frac{[\mathbf{M}, \mathbf{P}, \mathbf{Q}]}{\rho_{2}}, \Xi_{22}=\frac{\left(P_{2} Q_{0}^{h_{1}+h_{2}}-P_{0}^{h_{1}+h_{2}} Q_{2}\right) M_{1}+\left(P_{0}^{h_{1}+h_{2}} Q_{1}-P_{1} Q_{0}^{h_{1}+h_{2}}\right) M_{2}}{\rho_{3}} \\
\Xi_{23}=-\frac{[\mathbf{P}, \mathbf{M}]}{\rho_{3}} \\
\Xi_{31}=\frac{\Theta_{1}\left(P_{2} Q_{0}^{h_{1}}-P_{0}^{h_{1}} Q_{2}\right)+\Theta_{2}\left(P_{0}^{h_{1}} Q_{1}-P_{1} Q_{0}^{h_{1}}\right)+\Lambda \Lambda_{3}[P, Q]}{\rho_{2}} \\
\Xi_{32}=\frac{\Theta_{1}\left(P_{2} Q_{0}^{h_{1}+h_{2}}-P_{0}^{h_{1}+h_{2}} Q_{2}\right)+\Theta_{2}\left(P_{0}^{h_{1}+h_{2}} Q_{1}-P_{1} Q_{0}^{h_{1}+h_{2}}\right)+\Omega_{3}[P, Q]}{\rho_{3}} \\
\Xi_{33}=\frac{-\Theta_{1} P_{2}+\Theta_{2} P_{1}}{\rho_{3}}
\end{gathered}
$$

According to the fluctuation-dissipation theorem, the power spectrum of thermal height fluctuations of the interface and the free surface is given by

$$
S_{z z}^{(i j)}(k, \omega)=\int d t<u_{z}^{(i)}(k, t) u_{z}^{(j)}(-k, 0)>e^{i \omega t}=2 k_{B} T \frac{\operatorname{Im}\left[\chi_{z z}^{(i j)}(k, \omega)\right]}{\omega} .
$$

The frequencies and damping rates can be obtained by the poles of $\chi_{z z}^{(i j)}(k, \omega)$ in the lower half of the complex $\omega$ plane. For highly viscous polymeric liquids, propagating modes are usually suppressed, and only overdamped modes exist. The Fourier transform of the surface or interfacial fluctuation spectrum in the time domain is given as the sum of the overdamped modes at these poles as 


$$
S_{z z}^{(i j)}(k, t)=S_{z z}^{(i j)}(k) \sum_{m} a_{m}(k) e^{-\Gamma_{m}(k) t},
$$

where $S_{z z}^{(i j)}(k)=k_{B} T \chi_{z z}^{(i j)}(k, 0)$ is the total power of the spectrum and $a_{m}$ is the mode amplitude,

$$
a_{m}(k)=\frac{\operatorname{Res}\left[\chi_{z z}^{(i j)}\left(k,-i \Gamma_{m}\right)\right]}{\chi_{z z}^{(i j)}(k, 0) \Gamma_{m}}
$$

with the total amplitudes $\sum_{m} a_{m}(k)=1$.

The relaxation rates of free surface fluctuations of three layer thin films are numerically obtained by looking for the poles of $\chi_{z z}^{(33)}(k, \omega)$. 


\section{References}

1. Muller-Buschbaum P. Journal of Physics-Condensed Matter 2003;15(36):R1549-R1582.

2. Rauscher M and Dietrich S. Annual Review of Materials Research 2008;38(1):143-172.

3. Xue L and Han Y. Progress in Polymer Science 2011;36(2):269-293.

4. Xue L and Han Y. Progress in Materials Science 2012;57(6):947-979.

5. Hosaka N, Torikai N, Otsuka H, and Takahara A. Langmuir 2007;23(2):902-907.

6. Hosaka N, Otsuka H, Hino M, and Takahara A. Langmuir 2008;24(11):5766-5772.

7. Miyamoto K, Hosaka N, Kobayashi M, Otsuka H, Yamada N, Torikai N, and Takahara A. Polymer Journal 2007;39(12):1247-1252.

8. Harden JL, Pleiner H, and Pincus PA. The Journal of Chemical Physics 1991;94(7):5208.

9. Jackle J. Journal of Physics-Condensed Matter 1998;10(32):7121-7131.

10. Langevin D. Light scattering by liquid surfaces and complementary techniques. New York: M. Dekker, 1992.

11. Hoshino T, Ohmasa Y, Osada R, and Yao M. Physical Review E 2008;78(6).

12. Kim H, Rühm A, Lurio LB, Basu JK, Lal J, Lumma D, Mochrie SGJ, and Sinha SK. Physical Review Letters 2003;90(6).

13. Madsen A, Seydel T, Sprung M, Gutt C, Tolan M, and Grubel G. Phys Rev Lett 2004;92(9):096104.

14. Jiang Z, Kim H, Jiao X, Lee H, Lee YJ, Byun Y, Song S, Eom D, Li C, Rafailovich MH, Lurio LB, and Sinha SK. Physical Review Letters 2007;98(22).

15. Akgun B, Uğur Gke, Jiang Z, Narayanan S, Song S, Lee H, Brittain WJ, Kim H, Sinha SK, and Foster MD. Macromolecules 2009;42(3):737-741.

16. Wang S-f, Yang S, Lee J, Akgun B, Wu DT, and Foster MD. Physical Review Letters $2013 ; 111(6)$.

17. Sinha SK, Jiang Z, and Lurio LB. Adv Mater 2014;26(46):7764-7785.

18. Lee H, Li X, Kim H, Jiang Z, Lim J-R, and Lee J. Journal of the Korean Physical Society 2010;57(6):1412.

19. Jiang Z, Kim H, Mochrie SGJ, Lurio LB, and Sinha SK. Physical Review E 2006;74(1).

20. Yabashi M, Mochizuki T, Yamazaki H, Goto S, Ohashi H, Takeshita K, Ohata T, Matsushita T, Tamasaku K, Tanaka Y, and Ishikawa T. Nuclear Instruments \& Methods in Physics Research Section a-Accelerators Spectrometers Detectors and Associated Equipment 2001;467:678-681.

21. Hoshino T, Kikuchi M, Murakami D, Harada Y, Mitamura K, Ito K, Tanaka Y, Sasaki S, Takata M, Jinnai H, and Takahara A. J Synchrotron Radiat 2012;19(Pt 6):988-993.

22. Hoshino T, Murakami D, Ito K, Tanaka Y, Sasaki S, Takata M, Jinnai H, and Takahara A. Polymer Journal 2012;45(1):94-99. 
23. Hoshino T, Murakami D, Tanaka Y, Takata M, Jinnai H, and Takahara A. Phys Rev E Stat Nonlin Soft Matter Phys 2013;88(3):032602.

24. Tariq M, Serro AP, Mata JL, Saramago B, Esperança JMSS, Canongia Lopes JN, and Rebelo LPN. Fluid Phase Equilibria 2010;294(1-2):131-138.

25. Miyayama T, Sanada N, Suzuki M, Hammond JS, Si SQD, and Takahara A. Journal of Vacuum Science \& Technology A: Vacuum, Surfaces, and Films 2010;28(2):L1.

26. Yamamoto Y, Ichiki K, Seki T, Aoki T, and Matsuo J. Surface and Interface Analysis 2013;45(1):167-170.

27. Fujisawa T, Inoue K, Oka T, Iwamoto H, Uruga T, Kumasaka T, Inoko Y, Yagi N, Yamamoto M, and Ueki T. Journal of Applied Crystallography 2000;33(1):797-800.

28. Perlich J, Korstgens V, Metwalli E, Schulz L, Georgii R, and Muller-Buschbaum P. Macromolecules 2009;42(1):337-344.

29. Vrij A and Overbeek JT. Journal of the American Chemical Society 1968;90(12):3074-+.

30. Wyart FB and Daillant J. Canadian Journal of Physics 1990;68(9):1084-1088.

31. Xie R, Karim A, Douglas JF, Han CC, and Weiss RA. Physical Review Letters 1998;81(6):1251-1254.

32. Tolan M, Seeck OH, Schlomka JP, Press W, Wang J, Sinha SK, Li Z, Rafailovich MH, and Sokolov J. Physical Review Letters 1998;81(13):2731-2734.

33. Jiang Z, Mukhopadhyay MK, Song S, Narayanan S, Lurio LB, Kim H, and Sinha SK. Physical Review Letters 2008;101(24).

34. Sikorski M, Gutt C, Chushkin Y, Lippmann M, and Franz H. Phys Rev Lett 2010;105(21):215701. 
(a)

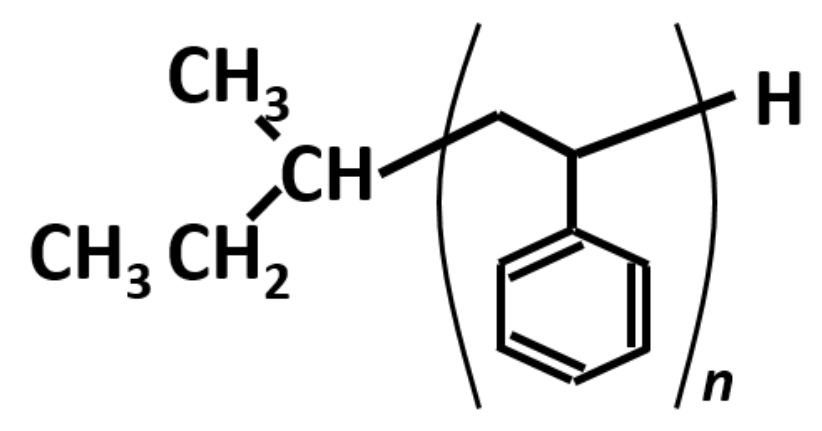

(b)

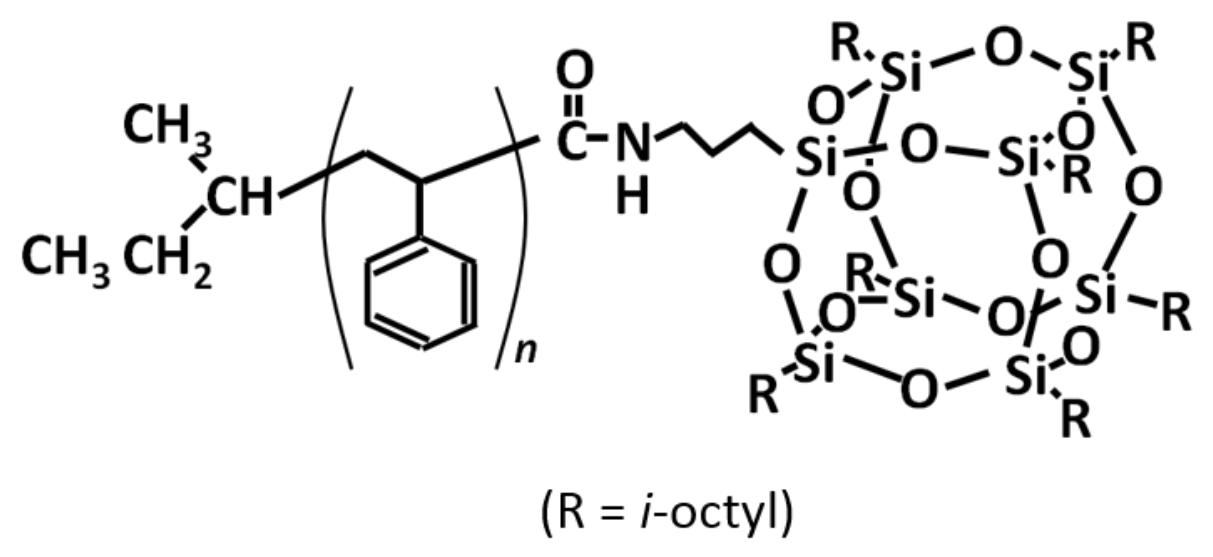

Figure 1. Chemical structures of (a) PS and (b) PS-POSS. 


\begin{tabular}{lccc}
\hline \multicolumn{1}{c}{ Sample } & \multicolumn{1}{c}{$M_{\mathrm{n}}$} & $M_{\mathrm{w}} / M_{\mathrm{n}}$ & $\begin{array}{c}\text { POSS content } \\
(\mathrm{wt} \%)\end{array}$ \\
\hline PS40k & 39500 & 1.04 & 0 \\
PS-POSS40k & 39500 & 1.04 & 3.0 \\
PS25k & 25000 & 1.06 & 0 \\
PS-POSS25k & 25000 & 1.06 & 4.6 \\
PS2.3k & 2300 & 1.21 & 0 \\
PS-POSS2.3k & 2300 & 1.21 & 33.6 \\
\hline
\end{tabular}

Table 1. Summary of molecular weights of PS and PS-POSS

\begin{tabular}{lccc}
\hline \multicolumn{1}{c}{ Sample } & $\eta($ Pa s) (at 413 K) & $\gamma\left(\mathrm{mN} \mathrm{m}^{-1}\right)($ at $413 \mathrm{~K})$ & $T_{\mathrm{g}}(\mathrm{K})$ \\
\hline PS40k & $2.25 \times 10^{5}$ & 18.7 & 375 \\
PS-POSS40k & $2.21 \times 10^{5}$ & 17.7 & 375 \\
PS25k & $5.86 \times 10^{4}$ & 19.6 & 374 \\
PS-POSS25k & $5.75 \times 10^{4}$ & 17.4 & 374 \\
\hline
\end{tabular}

Table 2. $\eta, \gamma$, and $T_{\mathrm{g}}$ of PS40k, PS-POSS40k, PS25k, and PS-POSS25k

\begin{tabular}{lccc}
\hline \multicolumn{1}{c}{ Sample } & $\eta(\mathrm{Pa} \mathrm{s})($ at $393 \mathrm{~K})$ & $\gamma\left(\mathrm{mN} \mathrm{m}^{-1}\right)($ at $393 \mathrm{~K})$ & $T_{\mathrm{g}}(\mathrm{K})$ \\
\hline PS2.3k & $1.09 \times 10^{3}$ & 21.2 & 348 \\
PS-POSS2.3k & $1.10 \times 10^{3}$ & 12.8 & 348 \\
\hline
\end{tabular}

Table 3. $\eta, \gamma$, and $T_{\mathrm{g}}$ of PS2.3k and PS-POSS2.3k 


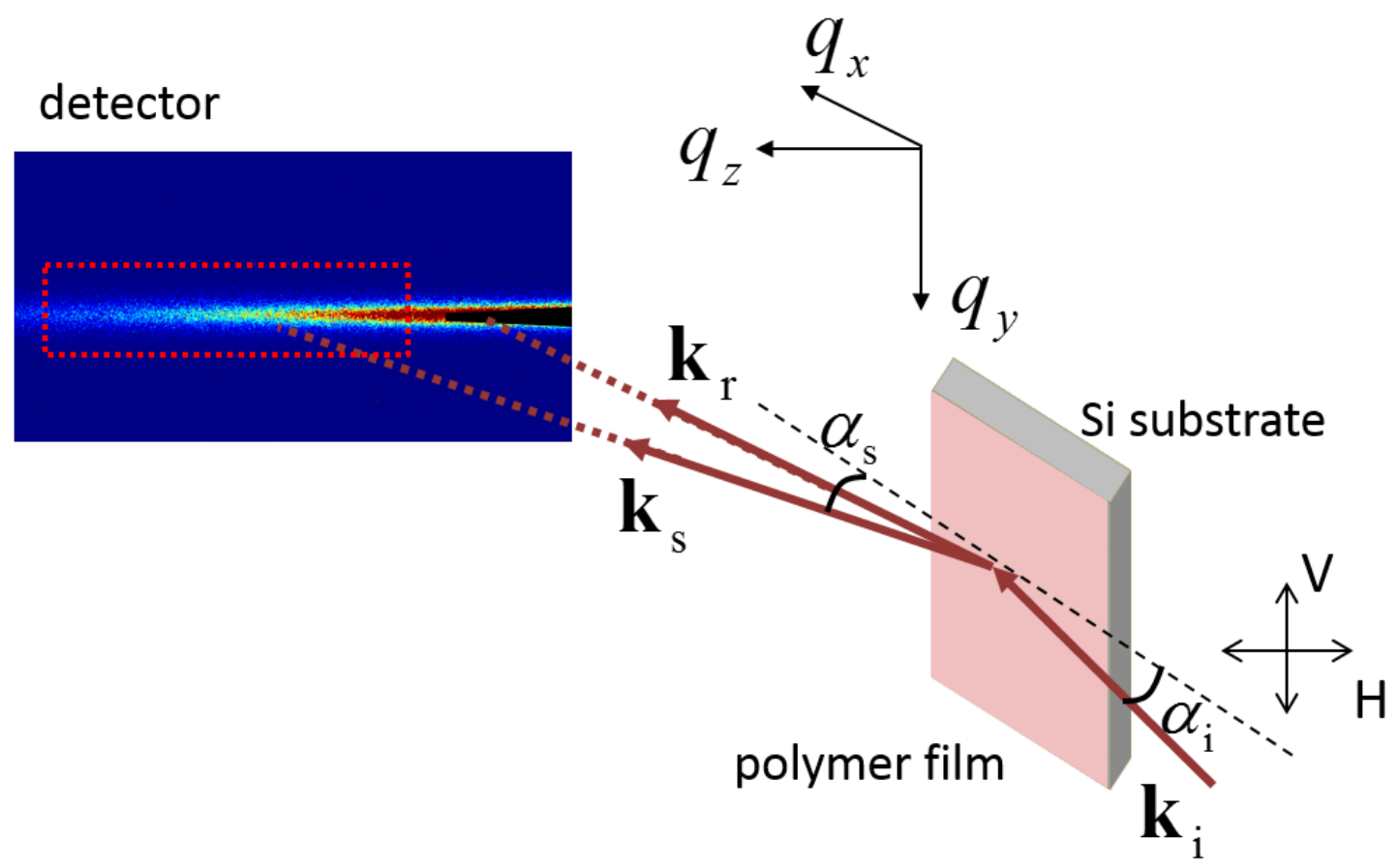

Figure 2 Schematic experimental setup for GI-XPCS. $\left(\alpha_{i}\right.$ and $\alpha_{\mathrm{s}}$ are the incident and the scattered angles, respectively.) The red box in the detector shows the ranges of the wave vectors analyzed for dynamics of surface fluctuations. 

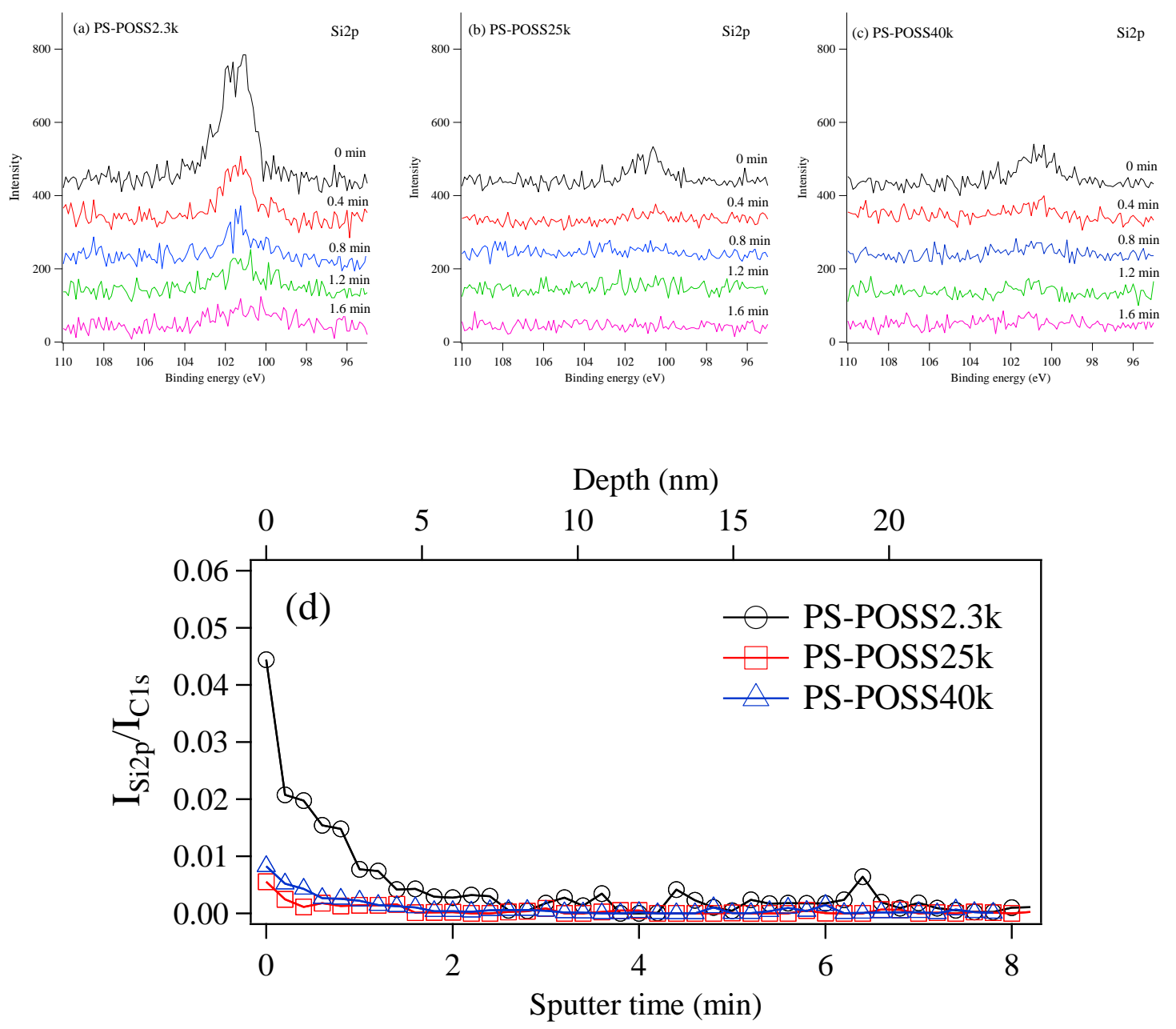

Figure 3 XPS spectra of Si 2p near surface of thin films of (a) PS-POSS2.3k, (b) PS-POSS25k and (c) PS-POSS40k sputtered by Ar gas cluster ion beam at various sputter time. (d) Depth profile of the signal ratio of Si2p to C1s for PS-POSS thin films. 

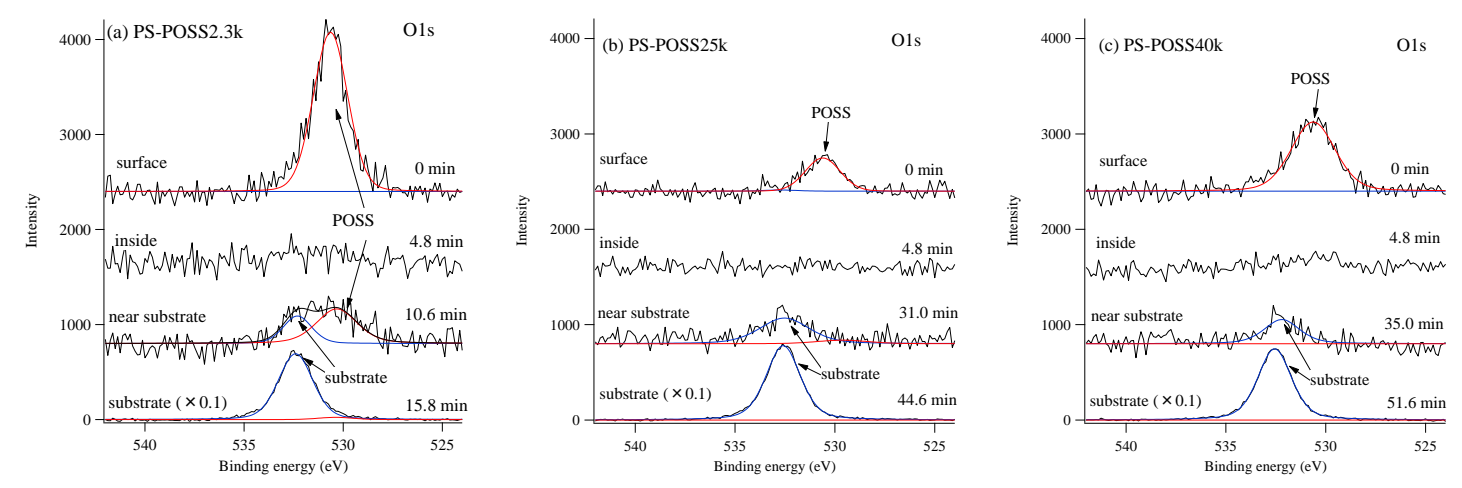

(d)

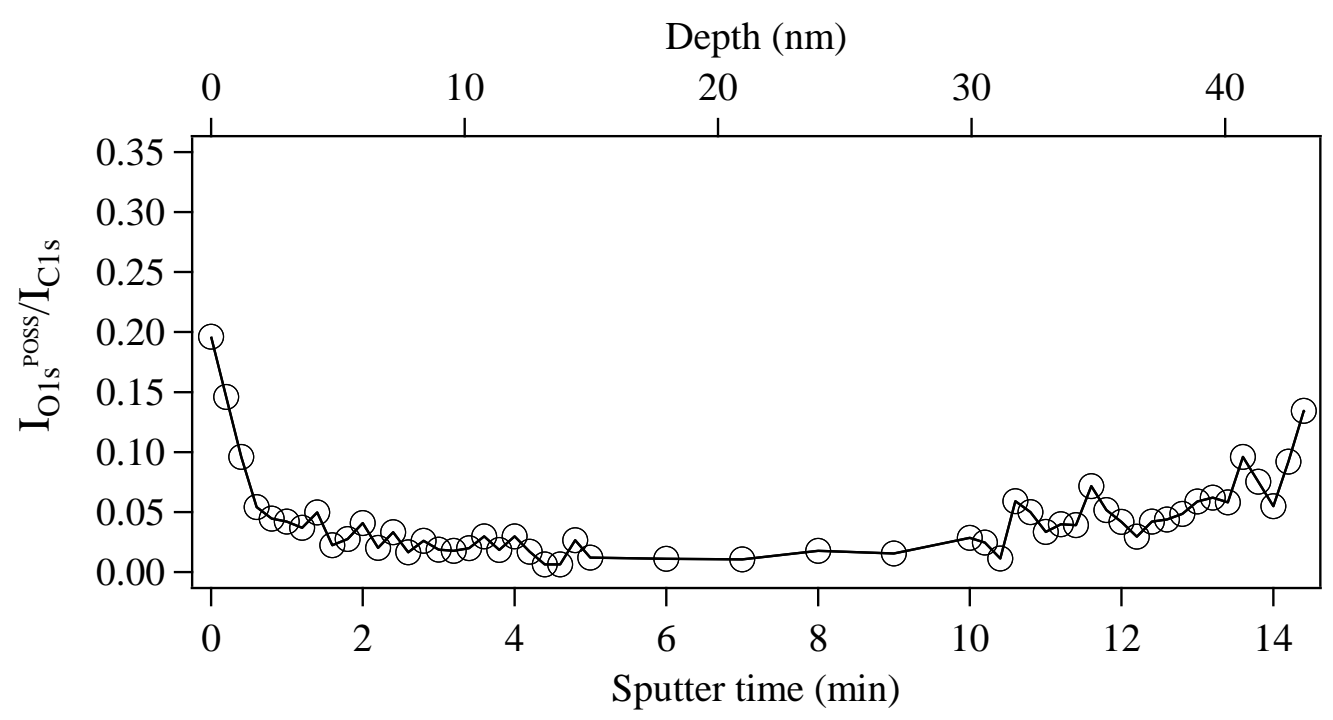

Figure 4 XPS spectra of O1s near surface of thin films of (a) PS-POSS2.3k, (b) PS-POSS25k and (c) PS-POSS40k sputtered by Ar gas cluster ion beam at various sputter time. Red lines and blue lines are fitting curve for POSS originating peak and substrate originating peak, respectively. (d) Depth profile of the signal ratio of POSS induced $\mathrm{O} 1 \mathrm{~s}$ to $\mathrm{C} 1 \mathrm{~s}$ for PS-POSS2.3k. 


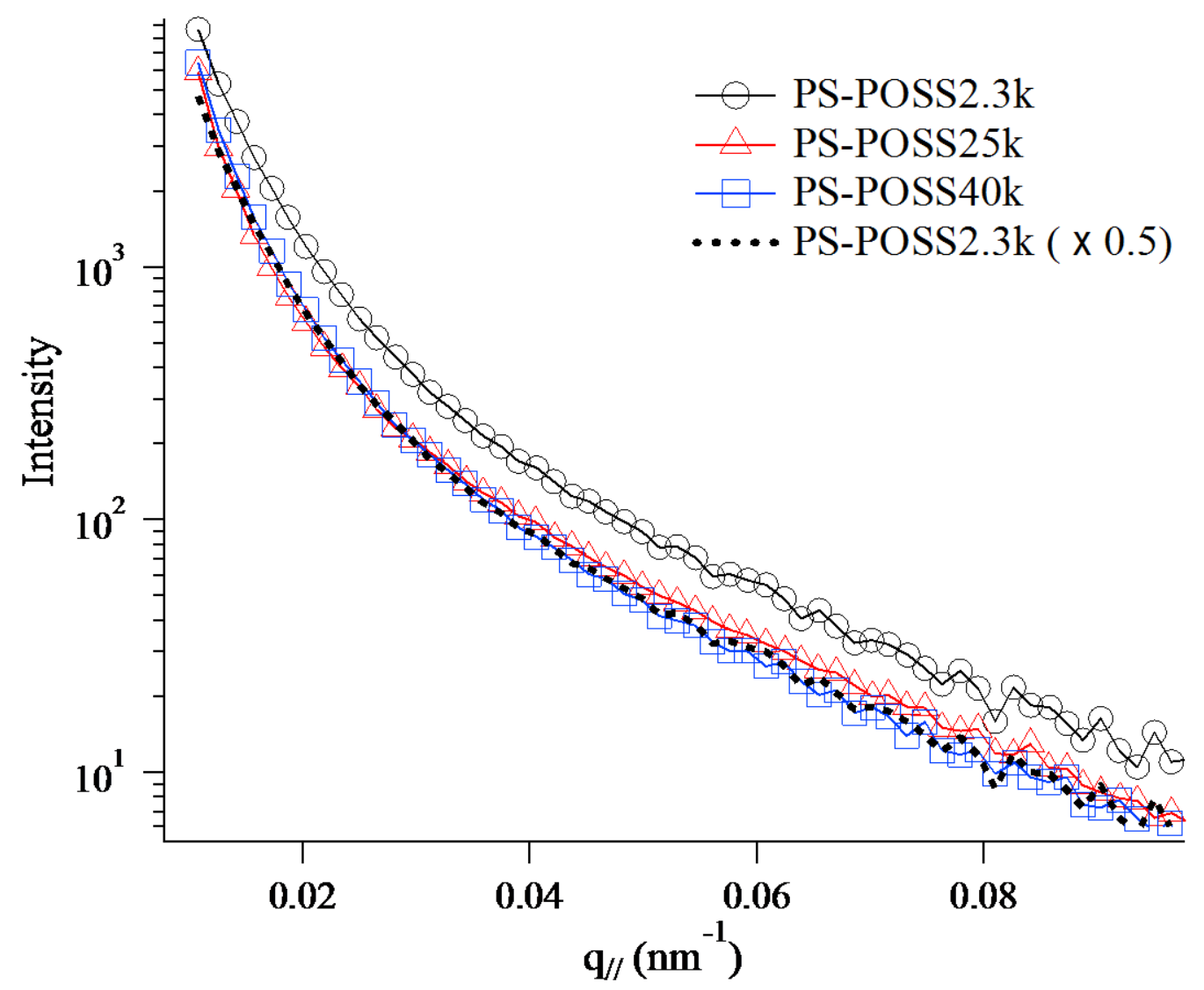

Figure 5 Scattering intensity profiles of PS-POSS2.3k, PS-POSS25k and PS-POSS40k thin films through grazing incidence small angle scattering (GI-SAXS) measurements. The dashed line is the intensity profile for PS-POSS2.3k multiplied by 0.5 . 
(a)

(b)

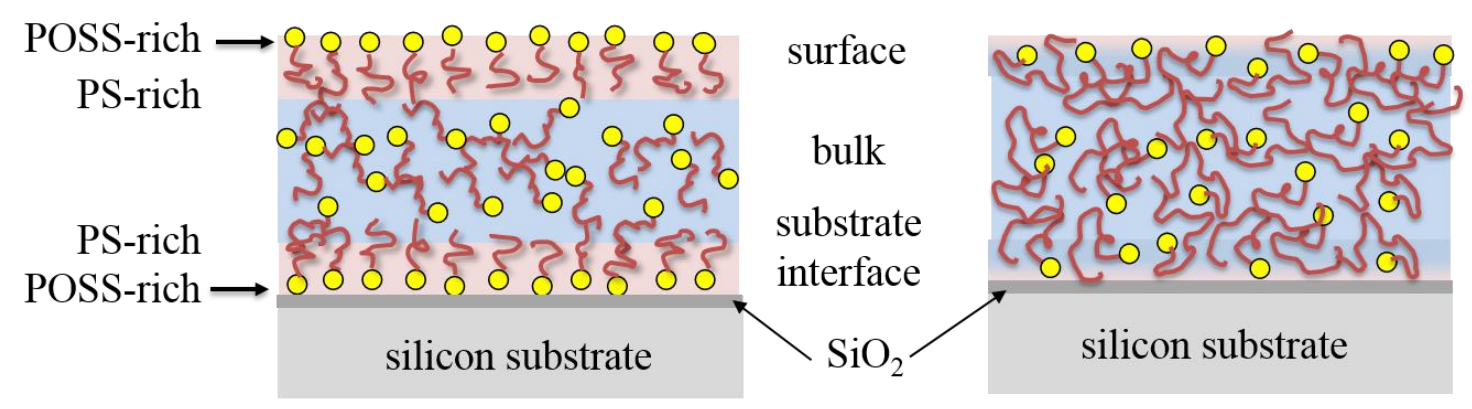

Figure 9. Schematic drawing of the structure of PS-POSS thin films for low molecular weight (a), and high molecular weight (b). 
(a)

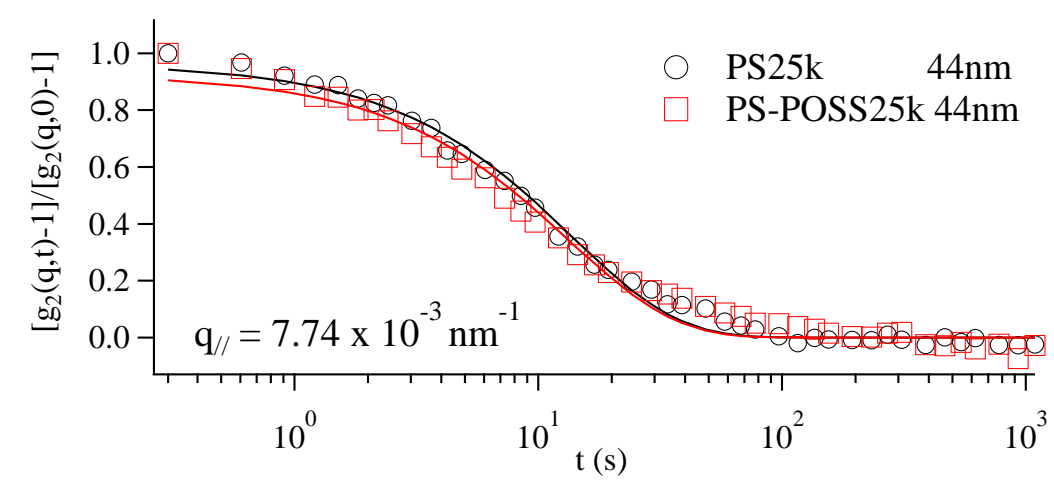

(b)

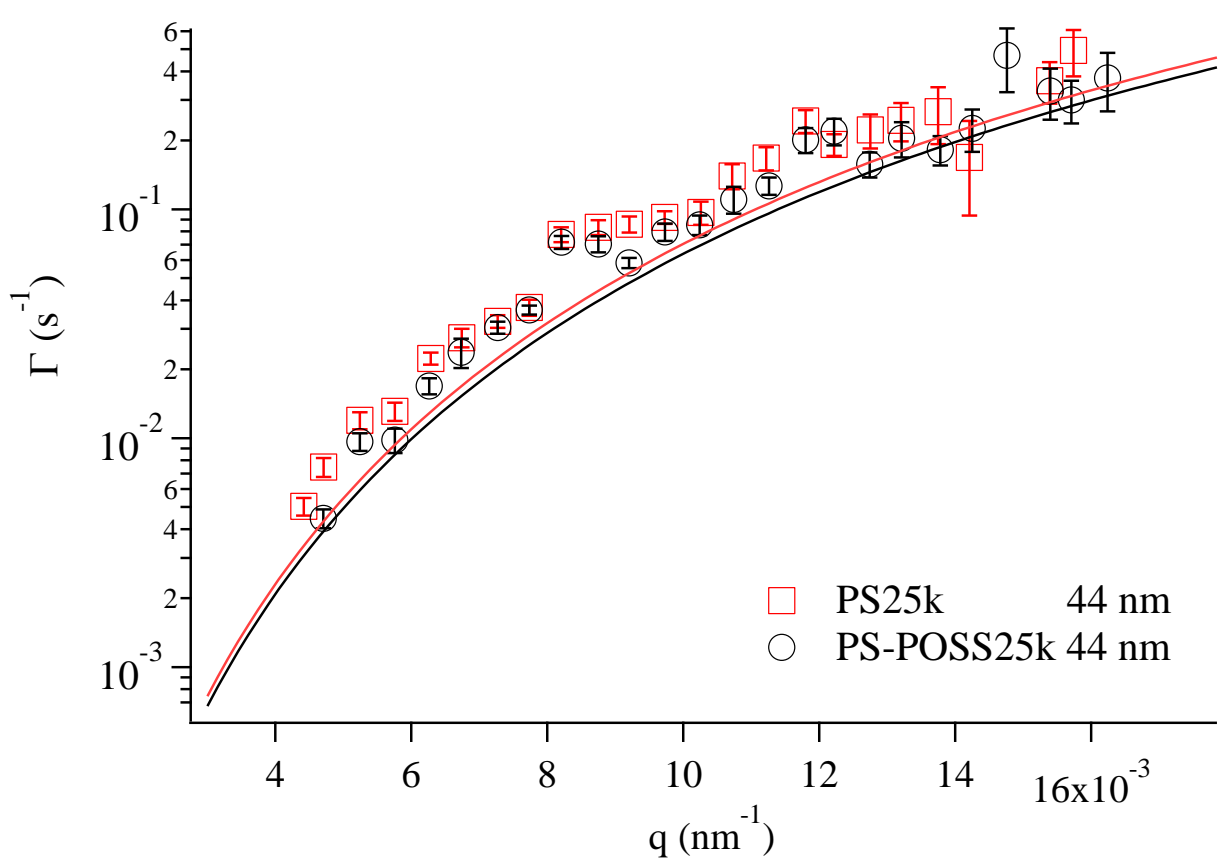


(c)

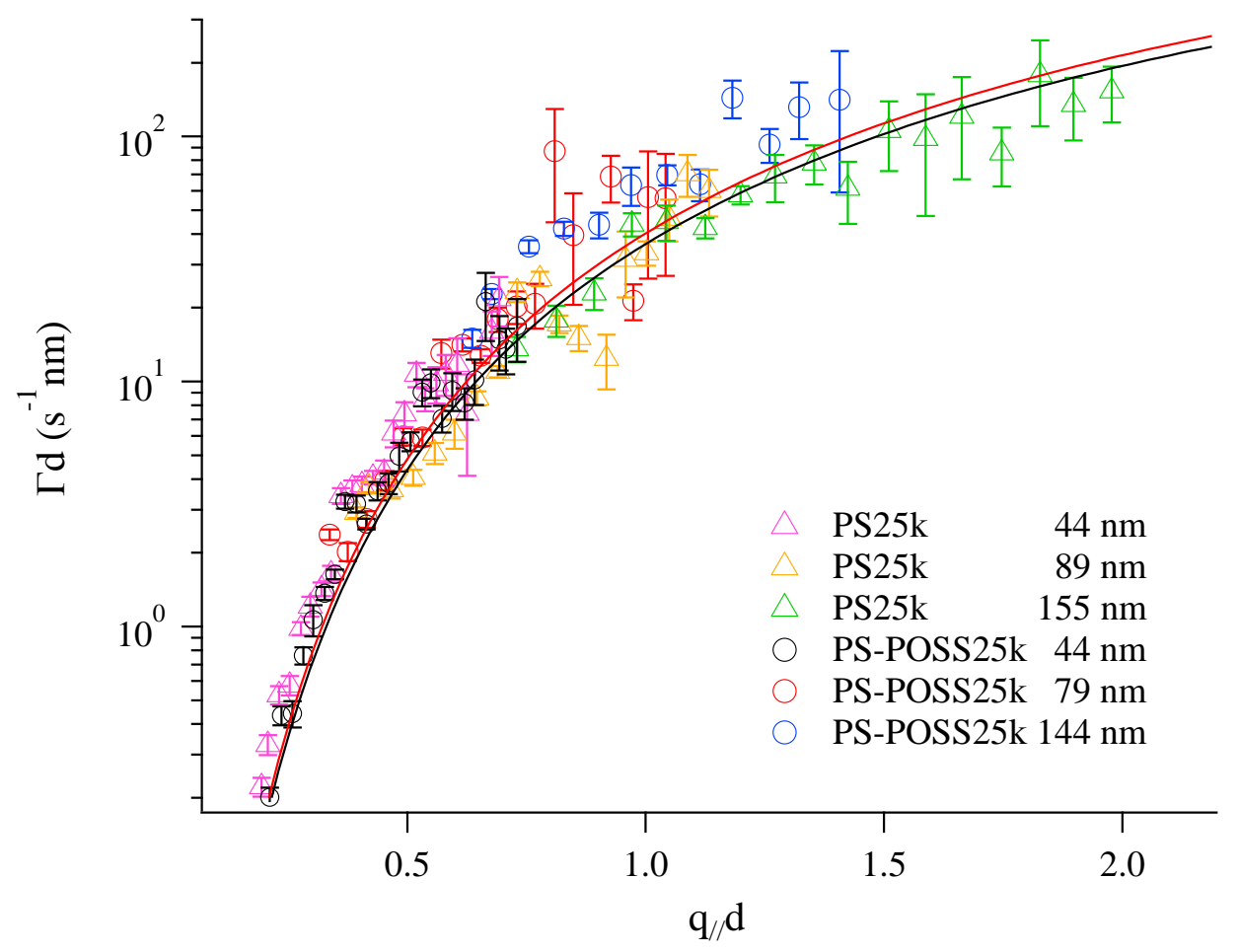

Figure 7. GI-XPCS results for PS25k and PS-POSS25k thin films at $413 \mathrm{~K}$. (a) Representative results of the measured normalized autocorrelation functions at $q_{/ /}=7.74$ $\times 10^{-3} \mathrm{~nm}^{-1}$ for 44-nm-thick thin films. The solid lines are fitting curves for Eq. (2). (b) $q_{/ /}$dependence of $\Gamma$ obtained from the fitting results of Eq. (2) for 44-nm-thick thin films. The red solid line and the black solid line are calculated from Eq. (3a) for PS25k and PS-POSS25k, respectively, with the values in Table 2. (c) $q_{/ /} d$ dependence of $\Gamma d$ for various thicknesses of thin films. The red solid line and the black line are calculated from Eq. (3b) for PS25k and PS-POSS25k, respectively, with the values in Table 2. 


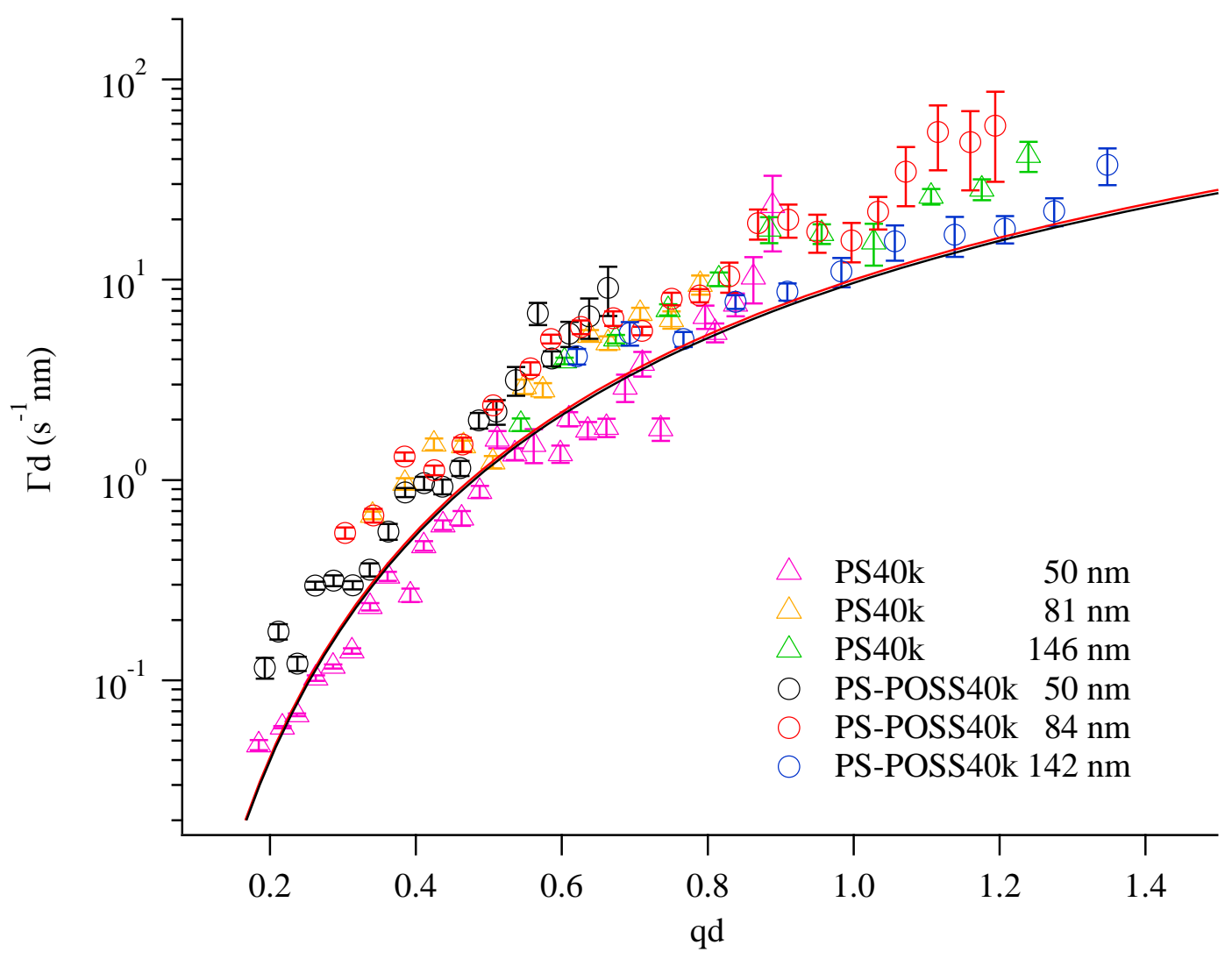

Figure 8. $q_{/ /} d$ dependence of $\Gamma d$ for various thicknesses of PS40k and PS-POSS40k thin films. The red solid line and the black line are calculated for PS40k and PS-POSS40k, respectively, from Eq. (3b) with the values in Table 2. 
(a)

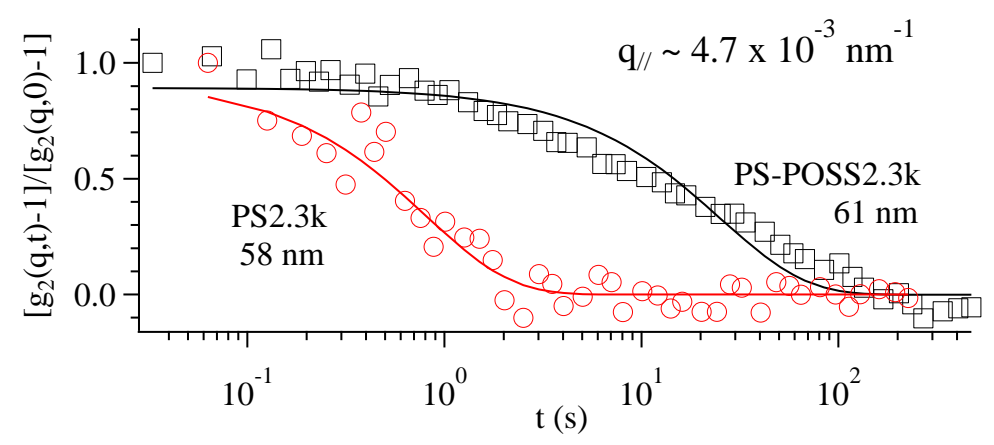

(b)

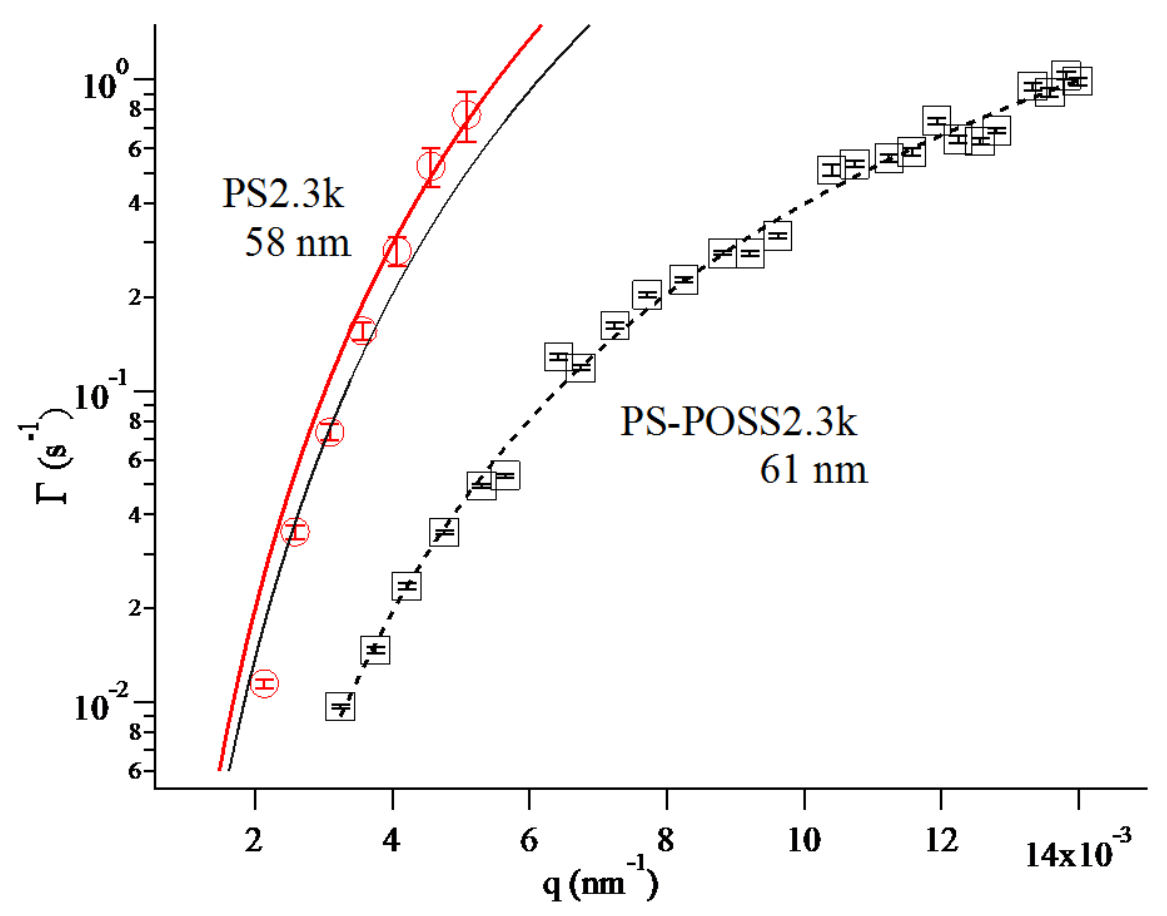

(c) 


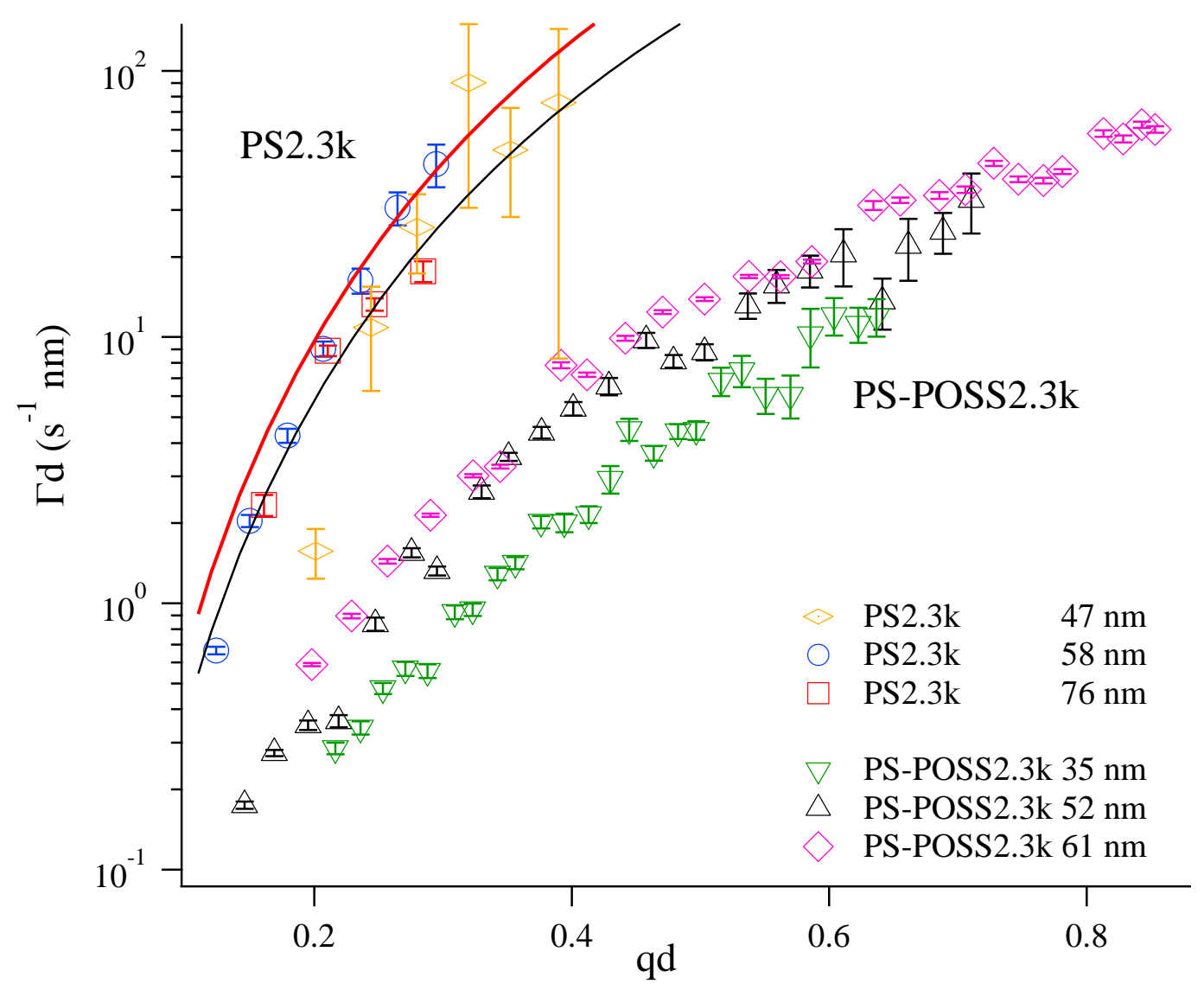

Figure 9. GI-XPCS results for 58-nm-thick PS2.3k thin film and 61-nm-thick PS-POSS2.3k thin film at $393 \mathrm{~K}$.

(a) Representative results of the measured normalized autocorrelation functions for 58-nm-thick PS2.3k thin film at $q_{/ /}=4.74 \times 10^{-3} \mathrm{~nm}^{-1}$ and for 61-nm-thick PS-POSS2.3k thin film at $q_{/ /}=4.76 \times 10^{-3} \mathrm{~nm}^{-1}$. The solid lines are fitting curves for Eq. (2).

(b) $q_{/ /}$dependence of $\Gamma$ obtained from the fitting results of Eq. (2) for 44-nm-thick thin films. The red solid line and the black solid line are calculated from Eq. (3a) for PS2.3k and PS-POSS2.3k, respectively, with the values in Table 3. The black dashed line is a visual guide.

(c) $q_{/ /} d$ dependence of $G d$ for various thicknesses of thin films. The red solid line and the black line are calculated from Eq. (3b) for PS2.3k and PS-POSS2.3k, respectively, with the values in Table 3 . 


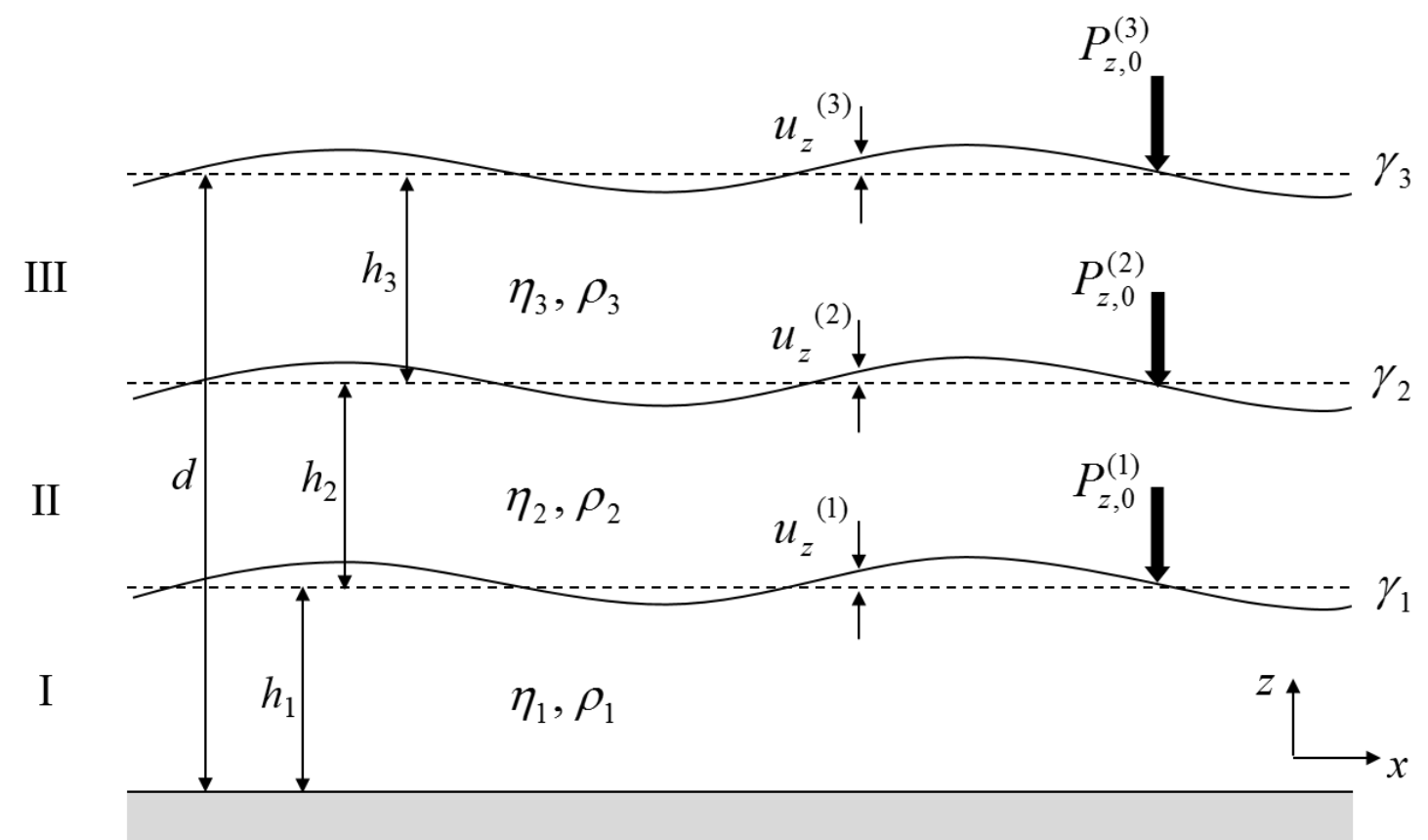

Figure 10. Geometry and notation of a supported three-layer film. The bottom, center, and top layers are denoted by regions I, II, and III, respectively. 


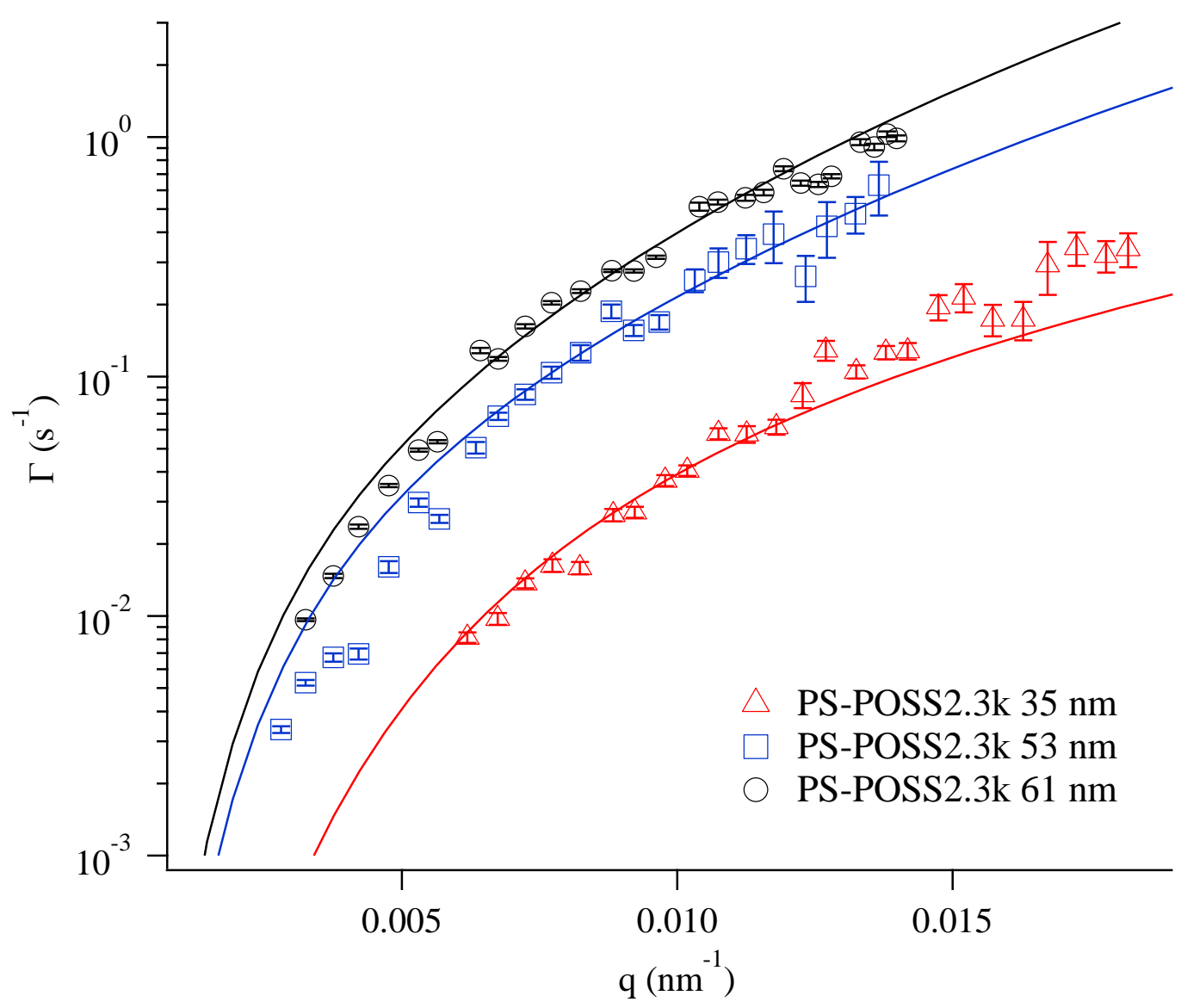

Figure 11. $q_{/ /}$dependence of $\Gamma$ obtained from the fitting results of Eq. (2) for 35, 53, and 61-nm-thick PS-POSS thin films. The solid lines are derived from the fluctuation-dissipation theorem for three-layer thin films with $\eta_{1}=\eta_{3}=9.6 \times 10^{4}$ $\mathrm{Pa} \cdot \mathrm{s}, \eta_{3}=1.10 \times 10^{3} \mathrm{~Pa} \cdot \mathrm{s}, d_{1}=16 \mathrm{~nm}, d_{3}=17 \mathrm{~nm}, \rho_{1}=\rho_{2}=\rho_{3}=1.0 \mathrm{~g} \cdot \mathrm{cm}^{-3}, \gamma_{1}=\gamma_{2}=0$, and $\gamma_{3}=12.8 \mathrm{mN} \cdot \mathrm{m}^{-1}$. 


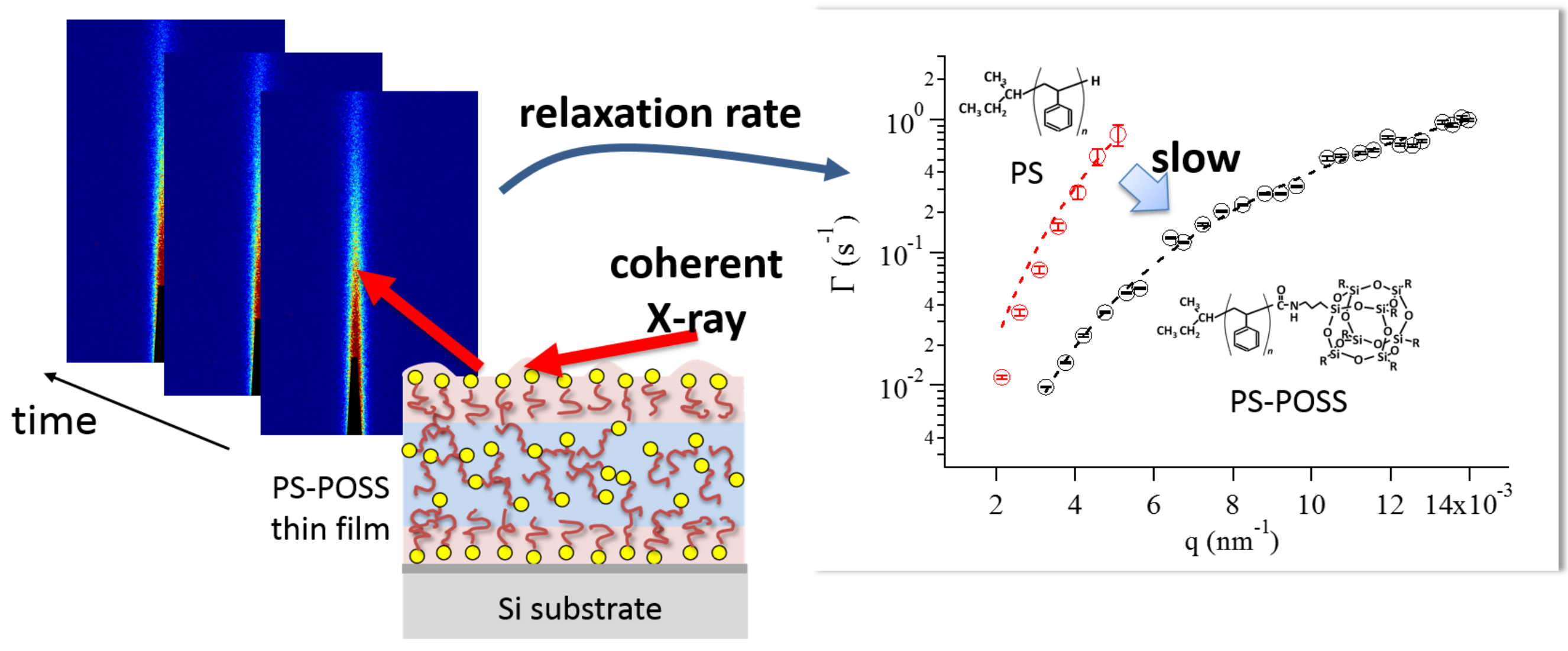

